\title{
Formation Tracking Control of Unicycle-Type Mobile Robots With Limited Sensing Ranges
}

\author{
K. D. Do
}

\begin{abstract}
A constructive method is presented to design cooperative controllers that force a group of $N$ unicycle-type mobile robots with limited sensing ranges to perform desired formation tracking and guarantee no collisions between the robots. Physical dimensions and dynamics of the robots are also considered in the control design. Smooth and $p$ times differential bump functions are introduced and incorporated into novel potential functions to design a formation tracking control system. Despite the robot limited sensing ranges, no switchings are needed to solve the collision avoidance problem. Simulations illustrate the results.
\end{abstract}

Index Terms-Bump function, formation tracking, mobile robot, potential function.

\section{INTRODUCTION}

$\mathbf{F}$ ORMATION control of multiple agents has received a lot of attention from the control community over the last few years due to its potential applications to search, rescue, coverage, surveillance, reconnaissance and cooperative transportation. Formation control can be roughly understood as controlling positions of a group of the agents such that they stabilize/ track desired locations relative to reference point(s), which can be another agent(s) within the team, and can either be stationary or moving. Research works on formation control often use one or more of leader-following (e.g., [1], [2]), behavioral (e.g., [3], [4]), and use of virtual structures (e.g., [5], [6]) approaches in either a centralized or decentralized manner. Centralized control schemes (e.g., [2] and [7]) use a single controller that generates collision-free trajectories in the workspace. Although these guarantee a complete solution, centralized schemes require high computational power and are not robust due to the heavy dependence on a single controller. A nice application of formation control based on the potential field method [2] and Lyapunov's direct method [8] to gradient climbing was recently addressed in [9]. However, the final configuration of formation cannot be foretold. On the other hand, decentralized schemes, see, e.g., [10] and [11], require less computational effort and are relatively more scalable to the team size. The decentralized approach usually involves a combination of agent-based local potential fields (e.g., [2], [12], and [13]). The main problem with the decentralized approach, when collision avoidance is taken into account, is that it is extremely difficult to predict and control the critical points of the controlled systems. An interesting work addressing

Manuscript received December 27, 2006; revised April 3, 2007. Manuscript received in final form June 17, 2007. Recommended by Associate Editor K. Kozlowski.

The author is with the School of Mechanical Engineering, The University of Western Australia, Crawley, WA 6009, Australia (e-mail: duc@mech.uwa.edu. au).

Color versions of one or more of the figures in this brief are available online at http://ieeexplore.ieee.org.

Digital Object Identifier 10.1109/TCST.2007.908214 geometric formation based on Voronoi partition optimization is given in [14], but the final arrangement of the agents cannot be foretold due to locality of Lloy's algorithm. Recently, a method based on a different navigation function from [15] provided a centralized formation stabilization control design strategy proposed in [7]. This work is extended to a decentralized version in [11]. A similar result but based on different navigation function motivated by the work in [15] is given in [16]. However, the formation is stabilized to any point in workspace instead of being "tied" to a fixed coordinate frame. Moreover, the potential function, which possesses all properties of a navigation function (see [15]), is finite when a collision occurs. This complicates the analysis of collision avoidance in the sense that one cannot directly use the first time derivative of the potential function to prove no collisions between agents. In [7], [15], [16], and [11], the tuning constants, which are crucial to guarantee that the only desired equilibrium points are asymptotically stable and that the other critical points are unstable, are extremely difficult to obtain explicitly. In most of the above papers, point-robots with simple (single or double integrator) dynamics (e.g., [2], [7], [11], [13], [14], and [17]) or fully actuated vehicles [6] (which can be converted to double-integrator dynamics via a feedback linearization) were investigated. It should be mentioned that decentralized navigation of nonpoint agents with single-integrator dynamics was also investigated in [18], but each agent requires global knowledge of position of other agents. Vehicles with nonholonomic constraints were also considered (e.g., [3]). However, the nonholonomic kinematics are transformed to double-integrator dynamics by controlling the hand position instead of the inertial position of the vehicles. Consequently, the vehicle heading is not controlled. In addition, switching control theory [19] is often used to design a decentralized formation control system (e.g., [1], where a case-by-case basis is proposed), especially when the vehicles have limited sensing ranges and collision avoidance between vehicles must be considered. Clearly, it is more desirable if we are able to design a nonswitching formation control system that can handle the above decentralized and collision avoidance requirements. Moreover, in the tracking control of single nonholonomic mobile robots (e.g., [20]-[22]), where it seems that the backstepping technique was first used in [20] to take the robot kinetic into account in the control design, the tracking errors are often interpreted in a frame attached to the reference trajectory using the coordinate transformation in [23] to overcome difficulties due to nonholonomic constraints. If these techniques are migrated to formation control of a group of mobile robots, it is extremely difficult to incorporate collision avoidance between the robots. The above problems motivate the contribution of this brief.

In this brief, cooperative controllers are designed to force a group of $N$ unicycle-type mobile robots with limited sensing ranges to perform desired formation tracking and to guarantee 


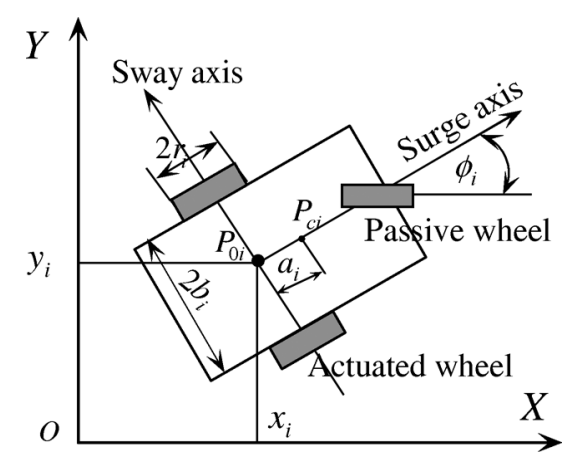

Fig. 1. Robot parameters.

no collisions between the robots. The physical dimensions and dynamics of the robots are also considered. The control development is based on novel potential functions, which attain the minimum value when the desired formation is achieved and are equal to infinity when a collision occurs. Moreover, smooth and $p$ times differential bump functions are introduced and incorporated into the potential functions to avoid the use of switchings, which are often required in literature to deal with the robot's limited sensing ranges.

\section{PRoblem Statement}

\section{A. Robot Dynamics}

We consider a group of $N$ mobile robots, of which each has the following dynamics [21]:

$$
\begin{aligned}
\dot{\eta}_{i} & =J_{i}\left(\eta_{i}\right) \omega_{i}, \\
M_{i} \dot{\omega}_{i} & =-C_{i}\left(\dot{\eta}_{i}\right) \omega_{i}-D_{i} \omega_{i}+\tau_{i}
\end{aligned}
$$

where $i=1, \ldots, N, \eta_{i}=\left[\begin{array}{lll}x_{i} & y_{i} & \phi_{i}\end{array}\right]^{T}$ denotes the position $\left(x_{i}, y_{i}\right)$, the coordinates of the middle point, $P_{0 i}$, between the left and right driving wheels, and heading $\phi_{i}$ of the robot $i$ coordinated in the earth-fixed frame $O X Y$ (see Fig. 1), $\omega_{i}=$ $\left[\omega_{1 i} \omega_{2 i}\right]^{T}$, where $\omega_{1 i}$ and $\omega_{2 i}$ are the angular velocities of the wheels of the robot $i, \tau_{i}=\left[\tau_{1 i} \tau_{2 i}\right]^{T}$, where $\tau_{1 i}$ and $\tau_{2 i}$ are the control torques applied to the wheels of the robot $i$. The rotation matrix $J_{i}\left(\eta_{i}\right)$, mass matrix $M_{i}$, Coriolis matrix $C_{i}\left(\dot{\eta}_{i}\right)$, and damping matrix $D_{i}$ in (1) are given by

$$
\begin{aligned}
J_{i}\left(\eta_{i}\right) & =\frac{r_{i}}{2}\left[\begin{array}{cc}
\cos \left(\phi_{i}\right) & \cos \left(\phi_{i}\right) \\
\sin \left(\phi_{i}\right) & \sin \left(\phi_{i}\right) \\
\frac{1}{b_{i}} & -\frac{1}{b_{i}}
\end{array}\right] \\
M_{i} & =\left[\begin{array}{cc}
m_{11 i} & m_{12 i} \\
m_{12 i} & m_{11 i}
\end{array}\right] \\
C_{i}\left(\dot{\eta}_{i}\right) & =\left[\begin{array}{cc}
0 & c_{i} \dot{\phi}_{i} \\
-c_{i} \dot{\phi}_{i} & 0
\end{array}\right] \\
D_{i} & =\left[\begin{array}{cc}
d_{11 i} & 0 \\
0 & d_{22 i}
\end{array}\right]
\end{aligned}
$$

with

$$
\begin{aligned}
c_{i} & =\frac{1}{2 b_{i}} r_{i}^{2} m_{c i} a_{i} \\
m_{11 i} & =\frac{1}{4 b_{i}^{2}} r_{i}^{2}\left(m_{i} b_{i}^{2}+I_{i}\right)+I_{w i} \\
m_{12 i} & =\frac{1}{4 b_{i}^{2}} r_{i}^{2}\left(m_{i} b_{i}^{2}-I_{i}\right) \\
m_{i} & =m_{c i}+2 m_{w i} \\
I_{i} & =m_{c i} a_{i}^{2}+2 m_{w i} b_{i}^{2}+I_{c i}+2 I_{m i}
\end{aligned}
$$

where $m_{c i}$ and $m_{w i}$ are the masses of the body and wheel with a motor, $I_{c i}, I_{w i}$, and $I_{m i}$ are the moments of inertia of the body about the vertical axis through $P_{c i}$ (center of mass), the wheel with the rotor of a motor about the wheel axis, and the wheel with the rotor of a motor about the wheel diameter, respectively, $r_{i}, a_{i}$, and $b_{i}$ are defined in Fig. 1, and the nonnegative constants $d_{11 i}$ and $d_{22 i}$ are the damping coefficients.

For convenience, we convert the wheel velocities $\left(\omega_{1 i}, \omega_{2 i}\right)$ of the robot $i$ to its linear $v_{i}$ and angular $w_{i}$ velocities by

$$
\varpi_{i}=B_{i}^{-1} \omega_{i} ; \quad B_{i}=\frac{1}{r_{i}}\left[\begin{array}{cc}
1 & b_{i} \\
1 & -b_{i}
\end{array}\right]
$$

where $\varpi_{i}=\left[v_{i} w_{i}\right]^{T}$, and $B_{i}$ is invertible since $\operatorname{det}\left(B_{i}\right)=$ $-2 b_{i} / r_{i}$. With (4), we can write the robot dynamics (1) as follows:

$$
\begin{aligned}
\dot{x}_{i} & =v_{i} \cos \left(\phi_{i}\right) \\
\dot{y}_{i} & =v_{i} \sin \left(\phi_{i}\right) \\
\dot{\phi}_{i} & =w_{i} \\
\bar{M}_{i} \dot{\varpi}_{i} & =-\bar{C}_{i}\left(w_{i}\right) \varpi_{i}-\bar{D}_{i} \varpi_{i}+\bar{B}_{i} \tau_{i}, \quad i=1, \ldots, N
\end{aligned}
$$

where

$$
\begin{aligned}
\bar{M}_{i} & =B_{i}^{-1} M_{i} B_{i}=\left[\begin{array}{cc}
\bar{m}_{11 i} & 0 \\
0 & \bar{m}_{22 i}
\end{array}\right] \\
\bar{C}_{i}\left(w_{i}\right) & =B_{i}^{-1} C_{i}\left(\dot{\eta}_{i}\right) B_{i}=\left[\begin{array}{cc}
0 & -b_{i} c_{i} w_{i} \\
\frac{c_{i}}{b_{i}} w_{i} & 0
\end{array}\right] \\
\bar{D}_{i} & =B_{i}^{-1} D_{i} B_{i}=\left[\begin{array}{ll}
\bar{d}_{11 i} & \bar{d}_{12 i} \\
\bar{d}_{21 i} & \bar{d}_{22 i}
\end{array}\right] \\
\bar{B}_{i} & =B_{i}^{-1}
\end{aligned}
$$

with

$$
\begin{aligned}
\bar{m}_{11 i} & =m_{11 i}+m_{12 i} \\
\bar{m}_{22 i} & =m_{11 i}-m_{12 i} \\
\bar{d}_{11 i} & =\frac{1}{2}\left(d_{11 i}+d_{22 i}\right) \\
\bar{d}_{12 i} & =\frac{b_{i}}{2}\left(d_{11 i}-d_{22 i}\right) \\
\bar{d}_{21 i} & =\frac{1}{2 b_{i}}\left(d_{11 i}-d_{22 i}\right) \\
\bar{d}_{22 i} & =\frac{1}{2}\left(d_{11 i}+d_{22 i}\right) .
\end{aligned}
$$




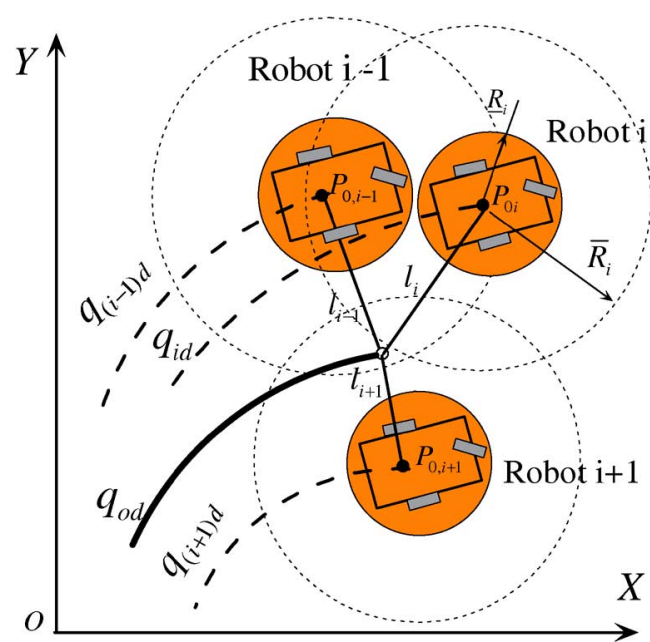

Fig. 2. Formation setup

\section{B. Formation Control Objective}

In this brief, we will study a formation control problem under the following assumption.

Assumption 1:

1) The robot $i$ has a physical safety circular area, which is centered at the point $P_{0 i}$, has a radius $\underline{R}_{i}$, and has a circular communication area, which is centered at the point $P_{0 i}$ and has a radius $\bar{R}_{i}$ (see Fig. 2). The radius $\bar{R}_{i}$ is strictly larger than $\underline{R}_{i}+\underline{R}_{j}, j=1, \ldots, N, j \neq i$.

2) The robot $i$ broadcasts its state $\left(x_{i}, y_{i}, \phi_{i}, \varpi_{i}\right)$ and its reference trajectory $q_{i d}$ in its communication area. Moreover, the robot $i$ can receive the states and reference trajectories broadcast by other robots $j, j=1, \ldots, N, j \neq i$, in the group if the points $P_{0 j}$ of these robots are in the communication area of the robot $i$.

3) The dimensional terms $\left(r_{i}, a_{i}, b_{i}\right)$ of the robot $i$ are known to the robot $i$. The terms involved with mass, inertia, and damping, $\left(m_{11 i}, m_{12 i}, d_{11 i}, d_{22 i}, c_{i}\right)$, of the robots are unknown but constant.

4) At the initial time $t_{0} \geq 0$, each robot starts at a location that is outside of the safety areas of other robots in the group, i.e., there exists a strictly positive constant $\varepsilon_{1}$ such that

$$
\left\|q_{i}\left(t_{0}\right)-q_{j}\left(t_{0}\right)\right\|-\left(\underline{R}_{i}+\underline{R}_{j}\right) \geq \varepsilon_{1}
$$

for all $(i, j) \in(1,2, \ldots, N), i \neq j$, where $q_{i}=\left[x_{i} y_{i}\right]^{T}$.

5) The reference trajectory for the robot $i$ is $q_{i d}=\left[x_{i d} y_{i d}\right]^{T}$, which is generated by

$$
q_{i d}=q_{o d}\left(s_{o d}\right)+l_{i}
$$

where $q_{\text {od }}\left(s_{\text {od }}\right)=\left[x_{\text {od }}\left(s_{\text {od }}\right) y_{\text {od }}\left(s_{\text {od }}\right)\right]^{T}$ is referred to as the common reference trajectory with $s_{o d}$ being the common trajectory parameter, and $l_{i}$ is a constant vector. The trajectory $q_{o d}$ satisfies the following conditions:

$$
\begin{aligned}
\lim _{t \rightarrow \infty} u_{o d}^{2}(t) & \neq 0 \\
u_{o d} & =\sqrt{x_{o d}^{\prime 2}+y_{o d}^{\prime 2}} \dot{s}_{o d} \\
\sqrt{x_{o d}^{\prime 2}+y_{o d}^{\prime 2}} & >0 \\
\left|u_{o d}(t)\right| & \leq u_{o d}^{\max }
\end{aligned}
$$

where $x_{o d}^{\prime}=\partial x_{o d} / \partial s_{o d}, y_{o d}^{\prime}=\partial y_{o d} / \partial s_{o d}$, and $u_{o d}^{\max }$ is a strictly positive constant. Moreover, $\dot{u}_{o d}$ and $\ddot{u}_{o d}$ are also bounded. The constant vectors $l_{i}, i=1,2, \ldots, N$, satisfy

$$
\left\|l_{i}-l_{j}\right\|-\left(\underline{R}_{i}+\underline{R}_{j}\right) \geq \varepsilon_{2}
$$

for all $(i, j) \in(1,2, \ldots, N), i \neq j$, where $\varepsilon_{2}$ is a strictly positive constant.

Remark 1: Items 1) and 2) in Assumption 1 specify the way each robot communicates with other robots in the group within its communication range. In Fig. 2, the robots $i$ and $i-1$ are communicating with each other since the points $P_{0, i-1}$ and $P_{0 i}$ are in the communication areas of the robots $i$ and $i-1$, respectively. The robots $i$ and $i+1$ are not communicating with each other since the points $P_{0 i}$ and $P_{0, i+1}$ are not in the communication areas of the robots $i+1$ and $i$, respectively. Similarly, the robots $i-1$ and $i+1$ are not communicating with each other. Item 3) makes sense in practice because the dimensional terms can be easily predetermined while the terms involving mass, inertia, and damping are often difficult to predetermine. Moreover, the assumptions in this item mean that the matrix $\bar{B}_{i}$ is known, while the matrices $\bar{M}_{i}$ and $\bar{D}_{i}$ and coefficients of the entries of the matrix $\bar{C}_{i}\left(w_{i}\right)$ are unknown but constant. In item 5), the constant vectors $l_{i}, i=1, \ldots, N$, specifies the desired formation configuration with respect to the earth-fixed frame $O X Y$. The condition (9) implies that the common reference $q_{\text {od }}$ is regular and its velocity $u_{o d}$, which specifies how the desired formation, whose configuration is determined by $l_{i}$, moves along $q_{o d}$, is bounded and satisfies a persistent excitation condition, i.e., the desired formation always moves along the common reference trajectory $q_{o d}$. The condition (10) specifies feasibility of the reference trajectories $q_{i d}, i=1,2, \ldots, N$ (recall from (8) that $q_{i d}-q_{j d}=l_{i}-l_{j}, \forall i \neq j$ ) due to physical safety circular areas of the robots. Finally, all of the robots in the group require knowledge of the common reference trajectory $q_{o d}$ since this trajectory specifies how the whole formation should move with respect to the earth-fixed frame $O X Y$.

Formation Control Objective: Under Assumption 1, design the control input $\tau_{i}$ and update laws for all terms involving mass, inertia, and damping $\left(m_{11 i}, m_{12 i}, d_{11 i}, d_{22 i}\right.$, and $\left.c_{i}\right)$ for each robot $i$ such that each robot asymptotically tracks its desired reference trajectory $q_{i d}$ while avoiding collisions with all other robots in the group, i.e., for all $(i, j) \in\{1,2, \ldots, N\}, i \neq j$, $t \geq t_{0} \geq 0$ :

$$
\begin{aligned}
\lim _{t \rightarrow \infty}\left(q_{i}(t)-q_{i d}(t)\right) & =0, \quad \lim _{t \rightarrow \infty}\left(\phi_{i}(t)-\phi_{i d}(t)\right)=0, \\
\left\|q_{i}(t)-q_{j}(t)\right\|-\left(\underline{R}_{i}+\underline{R}_{j}\right) & \geq \epsilon_{3}
\end{aligned}
$$

where $\phi_{i d}(t)=\arctan \left(y_{o d}^{\prime} / x_{o d}^{\prime}\right)$, and $\epsilon_{3}$ is a positive constant.

\section{PRELIMINARIES}

We here present one definition and one lemma, which will be used in the control design in the next section.

Definition 1: A scalar function $h(x, a, b)$ is called a $p$ times differential bump function if it enjoys the following properties

1) $h(x, a, b)=1, \quad$ if $\quad 0 \leq x \leq a$

2) $h(x, a, b)=0, \quad$ if $\quad x \geq b$

3) $0<h(x, a, b)<1$, if $a<x<b$

4) $h(x, a, b)$ is $p$ times differentiable with respect to $x$ 


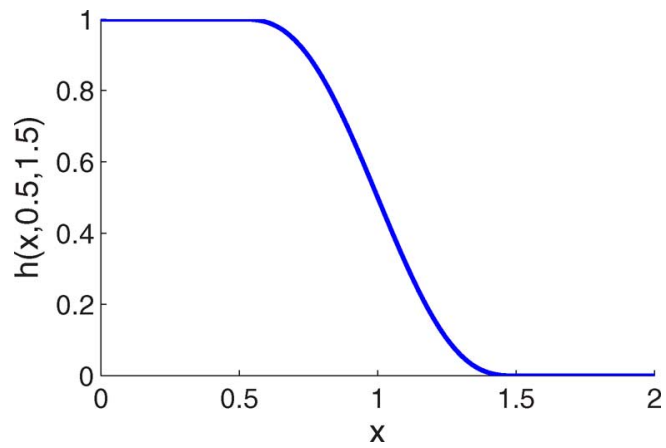

Fig. 3. Twice-differentiable bump function.

where $p$ is a positive integer, $x \in \mathbb{R}_{+}$, and $a$ and $b$ are constants such that $0 \leq a<b$. Moreover, if the function $h(x, a, b)$ is infinite times differentiable with respect to $x$, then it is called a smooth bump function.

It is noted that bump functions are not new to the control community. A nontrivial $C^{1}$ bump function was used in [24] for obstacle avoidance in flocking cooperation control of multiple agents with limited communication. Here, we give a constructive method to construct the $p$ times differentiable and smooth bump functions. These functions are useful when dealing with cooperative control of multiple agents with limited communication and high-order dynamics. Construction of these bump functions is given in the following lemma.

Lemma 1: Let the scalar function $h(x, a, b)$ be defined as

$$
h(x, a, b)=1-\frac{\int_{a}^{x} f(\tau-a) f(b-\tau) d \tau}{\int_{a}^{b} f(\tau-a) f(b-\tau) d \tau}
$$

where the function $f(y)$ is defined as follows:

$$
f(y)=0 \quad \text { if } y \leq 0 \text { and } f(y)=y^{p} \quad \text { if } y>0
$$

where $p$ is a positive integer. Then, the function $h(x, a, b)$ is a $p$ times differentiable bump function. Moreover, if $f(y)=y^{p}$ in (14) is replaced by $f(y)=e^{-1 / y}$, then property 4 ) is replaced by: $\left.4^{\prime}\right) h(x, a, b)$ is a smooth bump function.

Proof: See Appendix A. Fig. 3 illustrates a twice-differentiable bump function ( $a=0.5, b=1.5)$.

Remark 2: As specified in Lemma 1, the $p$ times differentiable bump functions can be obtained explicitly, i.e., the integral (13) can be solved analytically. The smooth bump functions cannot be obtained explicitly. Thus, a numerical procedure is needed to solve the integral (13). However, in many applications, including the formation control of mobile robots in this brief, the $p$ times differentiable bump functions are sufficient.

\section{Control Design}

Since the robot dynamics (5) are of a strict feedback form [25] with respect to the robot linear velocity $v_{i}$ and angular velocity $w_{i}$, we will use the backstepping technique [25] to design the control input $\tau_{i}$. The control design is divided into two main stages. In the first stage, we consider the first three equations of (5) with $v_{i}$ and $w_{i}$ being considered as immediate controls. In the second stage, the actual control $\tau_{i}$ will be designed.

\section{A. Stage I}

Since the robot is underactuated, we divide this stage into two steps using the backstepping technique. In the first step, the robot heading $\phi_{i}$ and the robot linear velocity $v_{i}$ are used as immediate controls to fulfill the task of position tracking and collision avoidance. In the second step, the robot angular velocity $w_{i}$ is used as an immediate control to stabilize the error between the actual robot heading and its immediate value at the origin. We do not use the transformation in [23] to interpret the tracking errors in a frame attached to the reference trajectories as is often done in literature (e.g., [20]-[22]) to avoid difficulties when dealing with collision avoidance.

1) Step I.1: Define

$$
\begin{aligned}
\phi_{i e} & =\phi_{i}-\alpha_{\phi_{i}} \\
v_{i e} & =v_{i}-\alpha_{v_{i}}
\end{aligned}
$$

where $\alpha_{\phi_{i}}$ and $\alpha_{v_{i}}$ are virtual controls of $\phi_{i}$ and $v_{i}$, respectively. With (15), the first two equations of (5) are read as

$$
\dot{q}_{i}=u_{i}+\Delta_{1 i}+\Delta_{2 i}
$$

where $q_{i}=\left[x_{i} y_{i}\right]^{T}$ and

$$
\begin{aligned}
u_{i} & =\left[\begin{array}{l}
\cos \left(\alpha_{\phi_{i}}\right) \\
\sin \left(\alpha_{\phi_{i}}\right)
\end{array}\right] \alpha_{v_{i}} \\
\Delta_{1 i} & =\left[\begin{array}{l}
\left(\cos \left(\phi_{i e}\right)-1\right) \cos \left(\alpha_{\phi_{i}}\right)-\sin \left(\phi_{i e}\right) \sin \left(\alpha_{\phi_{i}}\right) \\
\left(\sin \left(\phi_{i e}\right) \cos \left(\alpha_{\phi_{i}}\right)+\left(\cos \left(\phi_{i e}\right)-1\right) \sin \left(\alpha_{\phi_{i}}\right)\right.
\end{array}\right] \alpha_{v_{i}} \\
\Delta_{2 i} & =\left[\begin{array}{l}
\cos \left(\phi_{i}\right) \\
\sin \left(\phi_{i}\right)
\end{array}\right] v_{i e} .
\end{aligned}
$$

To fulfill the task of position tracking and collision avoidance, we consider the following potential function:

$$
\varphi_{I 1}=\sum_{i=1}^{N}\left(\gamma_{i}+0.5 \beta_{i}\right)
$$

where $\gamma_{i}$ and $\beta_{i}$ are the goal and related collision avoidance functions for the robot $i$ specified as follows.

- The goal function is designed such that it puts penalty on the tracking error for the robot and is equal to zero when the robot is at its desired position. A simple choice of this function is

$$
\gamma_{i}=0.5\left\|q_{i}-q_{i d}\right\|^{2} .
$$

- The related collision function $\beta_{i}$ should be chosen such that it is equal to infinity whenever any robots come in contact with the robot $i$, i.e., a collision occurs, and attains the minimum value when the robot $i$ is at its desired location with respect to other group members belonging to the set of $\mathbb{N}_{i}$ robots, where $\mathbb{N}_{i}$ is the set that contains all of the robots in the group except for the robot $i$. This function is chosen as follows:

$$
\beta_{i}=\sum_{j \in \mathbb{N}_{i}} \beta_{i j}
$$


where the function $\beta_{i j}=\beta_{j i}$ is a function of $\left\|q_{i j}\right\|^{2} / 2$ with $q_{i j}=q_{i}-q_{j}$ and enjoys the following properties:

1) $\beta_{i j}=0, \beta_{i j}^{\prime}=0$, and $\beta_{i j}^{\prime \prime} \geq 0$, if $\left\|q_{i j}\right\|=\left\|q_{i j d}\right\|$.

2) $\beta_{i j}>0$, if $0<\left\|q_{i j}\right\|<b_{i j}$.

3) $\beta_{i j}=0, \beta_{i j}^{\prime}=0, \beta_{i j}^{\prime \prime}=0$, and $\beta_{i j}^{\prime \prime \prime}=0$, if $\left\|q_{i j}\right\| \geq$ $b_{i j}$.

4) $\beta_{i j}=\infty$, if $\operatorname{Vertq} q_{i j} \| \leq\left(\underline{R}_{i}+\underline{R}_{j}\right)$.

5) $\beta_{i j} \leq \mu_{1},\left|\beta_{i j}^{\prime}\right| \leq \mu_{2}$, and $\left|\beta_{i j}^{\prime \prime} q_{i j}^{T} q_{i j}\right| \leq \mu_{3} \forall\left(\underline{R}_{i}+\right.$ $\left.\underline{R}_{j}\right)<\left\|q_{i j}\right\| \leq b_{i j}$.

6) $\beta_{i j}$ is at least three times differentiable with respect to

$$
\left\|q_{i j}\right\|^{2} / 2, \quad \text { if }\left\|q_{i j}\right\|>\left(\underline{R}_{i}+\underline{R}_{j}\right)
$$

where $q_{i j d}=q_{i d}-q_{j d}, b_{i j}$ is a strictly positive constant such that $\left(\underline{R}_{i}+\underline{R}_{j}\right)<b_{i j} \leq \min \left(\bar{R}_{i}, \bar{R}_{j},\left\|q_{i j d}\right\|\right), \mu_{1}$, $\mu_{2}$, and $\mu_{3}$ are positive constants, and $\beta_{i j}^{\prime}, \beta_{i j}^{\prime \prime}$, and $\beta_{i j}^{\prime \prime \prime}$ are defined as follows: $\beta_{i j}^{\prime}=\infty, \beta_{i j}^{\prime \prime}=\infty, \beta_{i j}^{\prime \prime \prime}=\infty$ if $\left\|q_{i j}\right\| \leq\left(\underline{R}_{i}+\underline{R}_{j}\right), \beta_{i j}^{\prime}=\partial \beta_{i j} / \partial\left(\left\|q_{i j}\right\|^{2} / 2\right), \beta_{i j}^{\prime \prime}=$ $\partial^{2} \beta_{i j} / \partial\left(\left\|q_{i j}\right\|^{2} / 2\right)^{2}$, and $\beta_{i j}^{\prime \prime \prime}=\left(\partial^{3} \beta_{i j} / \partial\left(\left\|q_{i j}\right\|^{2} / 2\right)^{3}\right.$ if $\left\|q_{i j}\right\|>\left(\underline{R}_{i}+\underline{R}_{j}\right)$.

Remark 3: Properties 1)-4) imply that the function $\beta_{i}$ is positive definite, is equal to zero when all of the robots are at their desired locations, and is equal to infinity when a collision between any robots in the group occurs. Moreover, Property 1) and the function $\gamma_{i}$ given in (19) ensure that the function $\varphi_{I 1}$ attains the (unique) minimum value of zero when all of the robots are at their desired positions. Also, Property 3 ) ensures that the collision avoidance between the robots $i$ and $j$ is only taken into account when they are in their communication areas. Property 5 ) is used to prove stability of the closed-loop system. Property 6) ensures that we can use techniques such as the backstepping and direct Lyapunov design methods [25], [26] for control design and stability analysis for continuous systems instead of techniques for switched, nonsmooth and discontinuous systems [19], [27] to handle the collision avoidance problem under the robot's limited sensing ranges.

There are many functions that satisfy all properties of $\beta_{i j}$ given in (21). An example is

$$
\beta_{i j}=\frac{h_{i j}\left(\left\|q_{i j}\right\|^{2} / 2, a_{i j}^{2} / 2, b_{i j}^{2} / 2\right)}{1-h_{i j}\left(\left\|q_{i j}\right\|^{2} / 2, a_{i j}^{2} / 2, b_{i j}^{2} / 2\right)}
$$

where $h_{i j}\left(\left\|q_{i j}\right\|^{2} / 2, a_{i j}^{2} / 2, b_{i j}^{2} / 2\right)$ is a $p$ times differentiable bump function defined in Definition 1 with $p \geq 3$ and $a_{i j} \geq\left(\underline{R}_{i}+\underline{R}_{j}\right)$, and $b_{i j} \leq \min \left(\bar{R}_{i}, \bar{R}_{j},\left\|q_{i j d}\right\|\right)$. The time derivative of $\varphi_{I 1}$ along the solutions of (16) satisfies

$$
\dot{\varphi}_{I 1}=\sum_{i=1}^{N} \Omega_{i}^{T}\left(u_{i}+\Delta_{1 i}+\Delta_{2 i}-\dot{q}_{o d}\right)
$$

where we have used $\dot{q}_{i d}=\dot{q}_{o d}, u_{i}-u_{j}=u_{i}-\dot{q}_{o d}-\left(u_{j}-\dot{q}_{o d}\right)$, $\forall(i, j) \in(1,2, \ldots, N), i \neq j$, and

$$
\Omega_{i}=q_{i}-q_{i d}+\sum_{j \in \mathbb{N}_{i}} \beta_{i j}^{\prime} q_{i j} .
$$

From (23), we choose a bounded control $u_{i}$ designed as follows:

$$
u_{i}=-k_{0} u_{o d}^{2} \Psi\left(\Omega_{i}\right)+\dot{q}_{o d}
$$

where $\Psi\left(\Omega_{i}\right)$ denotes a vector of bounded functions of elements of $\Omega_{i}$ in the sense that $\Psi\left(\Omega_{i}\right)=\left[\psi\left(\Omega_{i x}\right) \psi\left(\Omega_{i y}\right)\right]^{T}$ with $\Omega_{i x}$ and $\Omega_{i y}$ the first and second rows of $\Omega_{i}$, i.e., $\Omega_{i}=\left[\Omega_{i x} \Omega_{i y}\right]^{T}$. The function $\psi(x)$ is a scalar, at least three-times differentiable and bounded function with respect to $x$ and satisfies

1) $|\psi(x)| \leq \varrho_{1}$;

2) $\psi(x)=0$, if $x=0, x \psi(x)>0$, if $x \neq 0$;

3) $\psi(-x)=-\psi(x),(x-y)[\psi(x)-\psi(y)] \geq 0$;

4) $|\psi(x) / x| \leq \varrho_{2},|\partial \psi(x) / \partial x| \leq \varrho_{3}, \partial \psi(x) /\left.\partial x\right|_{x=0}=1$;

for all $x \in \mathbb{R}, y \in \mathbb{R}$, where $\varrho_{1}, \varrho_{2}, \varrho_{3}$ are strictly positive constants. Some functions that satisfy the above properties $\operatorname{are} \arctan (x)$ and $\tanh (x)$. The strictly positive constant $k_{0}$ is chosen such that

$$
k_{0}<\frac{1}{2 \varrho_{1} u_{o d}^{\max }} .
$$

The above condition ensures that $\alpha_{\phi_{i}}$ and $\alpha_{v_{i}}$ are solvable from $u_{i}$. We now need to solve for $\alpha_{\phi_{i}}$ and $\alpha_{v_{i}}$ from the expression of $u_{i}$ in (25) and (17). From (25) and (17), we have

$$
\begin{aligned}
& \cos \left(\alpha_{\phi_{i}}\right) \alpha_{v_{i}}=-k_{0} u_{o d}^{2} \psi\left(\Omega_{i x}\right)+\cos \left(\phi_{o d}\right) u_{o d} \\
& \sin \left(\alpha_{\phi_{i}}\right) \alpha_{v_{i}}=-k_{0} u_{o d}^{2} \psi\left(\Omega_{i y}\right)+\sin \left(\phi_{o d}\right) u_{o d}
\end{aligned}
$$

where we have used $\dot{x}_{o d}=x_{o d}^{\prime} \dot{s}_{o d}=$ $x_{o d}^{\prime} \sqrt{x_{o d}^{\prime 2}+y_{o d}^{\prime 2}} \dot{s}_{o d} / \sqrt{x_{o d}^{\prime 2}+y_{o d}^{\prime 2}}=\cos \left(\phi_{o d}\right) u_{o d}$ and $\dot{y}_{o d}=$ $y_{o d}^{\prime} \dot{s}_{o d}=y_{o d}^{\prime} \sqrt{x_{o d}^{\prime 2}+y_{o d}^{\prime 2}} \dot{s}_{o d} / \sqrt{x_{o d}^{\prime 2}+y_{o d}^{\prime 2}}=\sin \left(\phi_{o d}\right) u_{o d}$ since $\phi_{o d}=\arctan \left(y_{o d}^{\prime} / x_{o d}^{\prime}\right)$ and $\sqrt{x_{o d}^{\prime 2}+y_{o d}^{\prime 2}}>0$ (see Assumption 1). The left-hand sides of (27) are actually the coordinates of $u_{i}$ in the $x$ and $y$ directions. Now, multiplying both sides of the first equation of (27) with $\cos \left(\phi_{\text {od }}\right)$ and both sides of the second equation of (27) with $\sin \left(\phi_{o d}\right)$ and then adding the resulting equations together yields

$$
\begin{aligned}
\cos \left(\alpha_{\phi_{i}}-\phi_{o d}\right) \alpha_{v_{i}}=-k_{0} u_{o d}^{2} & \left(\psi\left(\Omega_{i x}\right) \cos \left(\phi_{o d}\right)\right. \\
& \left.+\psi\left(\Omega_{i x}\right) \sin \left(\phi_{o d}\right)\right)+u_{o d}
\end{aligned}
$$

On the other hand, multiplying both sides of the first equation of (27) with $\sin \left(\phi_{o d}\right)$ and both sides of the second equation of (27) with $\cos \left(\phi_{o d}\right)$ and then subtracting the resulting equations gives

$$
\begin{aligned}
& \sin \left(\alpha_{\phi_{i}}-\phi_{o d}\right) \alpha_{v_{i}} \\
& \quad=-k_{0} u_{o d}^{2}\left(-\psi\left(\Omega_{i x}\right) \sin \left(\phi_{o d}\right)+\psi\left(\Omega_{i x}\right) \cos \left(\phi_{o d}\right)\right) .
\end{aligned}
$$

From (28) and (29), we solve for $\alpha_{\phi_{i}}$ and $\alpha_{v_{i}}$ as shown in (30), shown at the bottom of the next page. It is noted that (30) is well defined since $-k_{0} u_{o d}\left(\psi\left(\Omega_{i x}\right) \cos \left(\phi_{o d}\right)+\right.$ $\left.\psi\left(\Omega_{i x}\right) \sin \left(\phi_{o d}\right)\right)+1 \geq-2 \varrho_{1} k_{0} u_{o d}^{\max }+1>0$, where the condition (26) has been used. Moreover, it is of interest to note that $\alpha_{\phi_{i}}$ and $\alpha_{v_{i}}$ are at least twice-differentiable functions of $q_{o d}, \phi_{o d}, u_{o d}, q_{i}$, and $q_{i j}$ with $j \in \mathbb{N}_{i}, j \neq i$.

Remark 4: When $\Omega_{i}$ defined in (24) is substituted into (25), the control $u_{i}$ can be written as

$u_{i}=k_{0} u_{o d}^{2}\left[\begin{array}{l}\psi\left(-\left(x_{i}-x_{i d}\right)-\sum_{j \in \mathbb{N}_{i}} \beta_{i j}^{\prime}\left(x_{i}-x_{j}\right)\right) \\ \psi\left(-\left(y_{i}-y_{i d}\right)-\sum_{j \in \mathbb{N}_{i}} \beta_{i j}^{\prime}\left(y_{i}-y_{j}\right)\right)\end{array}\right]+\dot{q}_{o d}$. 
It is seen from (31) that the argument of $\psi$ consists of two parts. The first part, $-\left(x_{i}-x_{i d}\right)$ or $-\left(y_{i}-y_{i d}\right)$, referred to as the attractive force, plays the role of forcing the robot $i$ to its desired location. The second part, $-\sum_{j \in \mathbb{N}_{i}} \beta_{i j}^{\prime}\left(x_{i}-x_{j}\right)$ or $-\sum_{j \in \mathbb{N}_{i}} \beta_{i j}^{\prime}\left(y_{i}-y_{j}\right)$, referred to as the repulsive force, takes care of collision avoidance for the robot $i$ with the other robots. Moreover, the immediate control $u_{i}$ of the robot $i$ given in (25) depends on only its own state and reference trajectory and the states of other neighbor robots $j$ if the points $P_{0 j}$ of these robots are in the circular communication area of the robot $i$, since outside this area $\beta_{i j}^{\prime}=0$ [see Property 3 ) of $\beta_{i j}$ ].

Now, substituting (25) into (23) results in

$$
\dot{\varphi}_{I 1}=-k_{0} u_{o d}^{2} \sum_{i=1}^{N} \Omega_{i}^{T} \Psi\left(\Omega_{i}\right)+\sum_{i=1}^{N} \Omega_{i}^{T}\left(\Delta_{1 i}+\Delta_{2 i}\right) .
$$

Substituting (25) into (16) results in

$$
\dot{q}_{i}=-k_{0} u_{o d}^{2} \Psi\left(\Omega_{i}\right)+\dot{q}_{o d}+\Delta_{1 i}+\Delta_{2 i} .
$$

2) Step I.2: In this step, we view $w_{i}$ as an immediate control to stabilize $\phi_{i e}$ at the origin. As such, we define

$$
w_{i e}=w_{i}-\alpha_{w_{i}}
$$

where $\alpha_{w_{i}}$ is a virtual control of $w_{i}$. To prepare for designing $\alpha_{w_{i}}$, let us calculate $\dot{\phi}_{i e}$. Differentiating both sides of the first equation of (15) along the solutions of (34), the third equation of (5) and the second equation of (30) results in

$$
\begin{aligned}
\dot{\phi}_{i e}= & w_{i e}+\alpha_{w_{i}}-\frac{\partial \alpha_{\phi_{i}}}{\partial q_{o d}} \dot{q}_{o d}-\frac{\partial \alpha_{\phi_{i}}}{\partial \phi_{o d}} \dot{\phi}_{o d}-\frac{\partial \alpha_{\phi_{i}}}{\partial u_{o d}} \dot{u}_{o d} \\
& -\frac{\partial \alpha_{\phi_{i}}}{\partial q_{i}}\left(u_{i}+\Delta_{1 i}+\Delta_{2 i}\right)-\sum_{j=1, j \neq i}^{N} \frac{\partial \alpha_{\phi_{i}}}{\partial q_{i j}} \\
& \times\left(u_{i}+\Delta_{1 i}+\Delta_{2 i}-\left(u_{j}+\Delta_{1 j}+\Delta_{2 j}\right)\right) .
\end{aligned}
$$

To design the virtual control $\alpha_{w_{i}}$, we consider the function

$$
\varphi_{I 2}=\varphi_{I 1}+0.5 \sum_{i=1}^{N} \phi_{i e}^{2}
$$

whose derivative along the solutions of (32) and (35) satisfies

$$
\begin{gathered}
\dot{\varphi}_{I 2}=-k_{0} u_{o d}^{2} \sum_{i=1}^{N} \Omega_{i}^{T} \Psi\left(\Omega_{i}\right)+\sum_{i=1}^{N} \Omega_{i}^{T} \Delta_{2 i}+\sum_{i=1}^{N} \phi_{i e} \\
\times\left(\frac{\Omega_{i}^{T} \Delta_{1 i}}{\phi_{i e}}+w_{i e}+\alpha_{w_{i}}-\frac{\partial \alpha_{\phi_{i}}}{\partial q_{o d}} \dot{q}_{o d}-\frac{\partial \alpha_{\phi_{i}}}{\partial \phi_{o d}} \dot{\phi}_{o d}\right. \\
-\frac{\partial \alpha_{\phi_{i}}}{\partial u_{o d}} \dot{u}_{o d}-\frac{\partial \alpha_{\phi_{i}}}{\partial q_{i}}\left(u_{i}+\Delta_{1 i}+\Delta_{2 i}\right) \\
-\sum_{j=1, j \neq i}^{N} \frac{\partial \alpha_{\phi_{i}}}{\partial q_{i j}}\left(u_{i}+\Delta_{1 i}+\Delta_{2 i}\right. \\
\left.\left.-\left(u_{j}+\Delta_{1 j}+\Delta_{2 j}\right)\right)\right)
\end{gathered}
$$

It is noted that $\Delta_{1 i} / \phi_{i e}$ is well defined since $\sin \left(\phi_{i e}\right) / \phi_{i e}=$ $\int_{0}^{1} \cos \left(\lambda \phi_{i e}\right) d \lambda$ and $\left(\cos \left(\phi_{i e}\right)-1\right) / \phi_{i e}=\int_{1}^{0} \sin \left(\lambda \phi_{i e}\right) d \lambda$ are smooth functions for all $\phi_{i e} \in \mathbb{R}$. From (37), we choose the virtual control $\alpha_{w_{i}}$ as

$$
\begin{aligned}
\alpha_{w_{i}}= & -k_{i} \phi_{i e}-\frac{\Omega_{i}^{T} \Delta_{1 i}}{\phi_{i e}}+\frac{\partial \alpha_{\phi_{i}}}{\partial q_{o d}} \dot{q}_{o d}+\frac{\partial \alpha_{\phi_{i}}}{\partial \phi_{o d}} \dot{\phi}_{o d} \\
& +\frac{\partial \alpha_{\phi_{i}}}{\partial u_{o d}} \dot{u}_{o d}+\frac{\partial \alpha_{\phi_{i}}}{\partial q_{i}}\left(u_{i}+\Delta_{1 i}\right) \\
& +\sum_{j=1, j \neq i}^{N} \frac{\partial \alpha_{\phi_{i}}}{\partial q_{i j}}\left(u_{i}+\Delta_{1 i}-\left(u_{j}+\Delta_{1 j}\right)\right)
\end{aligned}
$$

where $k_{i}$ is a positive constant. It is of interest to note that $\alpha_{w_{i}}$ is an at least once-differentiable function of $q_{o d}, \dot{q}_{o d}, \phi_{o d}, \dot{\phi}_{o d}$, $u_{o d}, \dot{u}_{o d}, q_{i}, \phi_{i}, q_{i j}$, and $\phi_{j}$, with $j \in \mathbb{N}_{i}, j \neq i$. Moreover, it should be noted that the virtual control $\alpha_{w_{i}}$ contains only the state and reference trajectory of the robot $i$, and the states of other neighbor robots $j$ if the points $P_{0 j}$ of these robots are in the communication area of the robot $i$, because outside this area $\partial \alpha_{\phi_{i}} / \partial q_{i j}=0$ thanks to Property 3) of $\beta_{i j}$. Substituting (38)

$$
\begin{aligned}
\alpha_{\phi_{i}} & =\phi_{o d}+\arctan \left(\frac{-k_{0} u_{o d}\left(-\psi\left(\Omega_{i x}\right) \sin \left(\phi_{o d}\right)+\psi\left(\Omega_{i x}\right) \cos \left(\phi_{o d}\right)\right)}{-k_{0} u_{o d}\left(\psi\left(\Omega_{i x}\right) \cos \left(\phi_{o d}\right)+\psi\left(\Omega_{i x}\right) \sin \left(\phi_{o d}\right)\right)+1}\right) \\
\alpha_{v_{i}} & =\cos \left(\alpha_{\phi_{i}}\right)\left(-k_{0} u_{o d}^{2} \psi\left(\Omega_{i x}\right)+\cos \left(\phi_{o d}\right) u_{o d}\right)+\sin \left(\alpha_{\phi_{i}}\right)\left(-k_{0} u_{o d}^{2} \psi\left(\Omega_{i y}\right)+\sin \left(\phi_{o d}\right) u_{o d}\right)
\end{aligned}
$$

$$
\dot{\varphi}_{I 2}=-k_{0} u_{o d}^{2} \sum_{i=1}^{N} \Omega_{i}^{T} \Psi\left(\Omega_{i}\right)-\sum_{i=1}^{N} k_{i} \phi_{i e}^{2}+\sum_{i=1}^{N}\left[\phi_{i e} w_{i e}+\left(\Omega_{i}^{T}-\phi_{i e} \frac{\partial \alpha_{\phi_{i}}}{\partial q_{i}}-\sum_{j=1, j \neq i}^{N}\left(\frac{\partial \alpha_{\phi_{i}}}{\partial q_{i j}} \phi_{i e}-\frac{\partial \alpha_{\phi_{j}}}{\partial q_{j i}} \phi_{j e}\right)\right) \Delta_{2 i}\right]
$$


into (37) results in (39), shown at the bottom of the previous page, after some manipulation. Substituting (38) into (35) gives

$$
\begin{aligned}
\dot{\phi}_{i e}= & -k_{i} \phi_{i e}-\frac{\Omega_{i}^{T} \Delta_{1 i}}{\phi_{i e}}-\frac{\partial \alpha_{\phi_{i}}}{\partial q_{i}} \Delta_{2 i} \\
& -\sum_{j=1, j \neq i}^{N} \frac{\partial \alpha_{\phi_{i}}}{\partial q_{i j}}\left(\Delta_{2 i}-\Delta_{2 j}\right)+w_{i e}
\end{aligned}
$$

To prepare for the next section, let us compute the term $\bar{M}_{i} \dot{\varpi}_{i e}$, where $\varpi_{i e}=\left[v_{i e} w_{i e}\right]^{T}$. From the second equation of (15), (34), and the last equation of (5), we have

$$
\begin{aligned}
\bar{M}_{i} \dot{\varpi}_{i e} & =-\bar{C}_{i}\left(w_{i}\right) \varpi_{i}-\bar{D}_{i} \varpi_{i}-\bar{M}_{i}\left[\dot{\alpha}_{v_{i}} \dot{\alpha}_{w_{i}}\right]^{T}+\bar{B}_{i} \tau_{i} \\
& =-\bar{D}_{i} \varpi_{i e}+\Phi_{i} \Theta_{i}+\bar{B}_{i} \tau_{i}
\end{aligned}
$$

where

$$
\begin{aligned}
& \dot{\alpha}_{v_{i}}=\frac{\partial \alpha_{v_{i}}}{\partial q_{o d}} \dot{q}_{o d}+\frac{\partial \alpha_{v_{i}}}{\partial u_{o d}} \dot{u}_{o d}+\frac{\partial \alpha_{v_{i}}}{\partial \phi_{o d}} \dot{\phi}_{o d}+\frac{\partial \alpha_{v_{i}}}{\partial q_{i}} \vartheta_{i} \\
& +\sum_{j=1, j \neq i}^{N} \frac{\partial \alpha_{v_{i}}}{\partial q_{i j}}\left(\vartheta_{i}-\vartheta_{j}\right) \\
& \dot{\alpha}_{w_{i}}=\frac{\partial \alpha_{w_{i}}}{\partial q_{o d}} \dot{q}_{o d}+\frac{\partial \alpha_{w_{i}}}{\partial \dot{q}_{o d}} \ddot{q}_{o d}+\frac{\partial \alpha_{w_{i}}}{\partial u_{o d}} \dot{u}_{o d}+\frac{\partial \alpha_{w_{i}}}{\partial \dot{u}_{o d}} \ddot{u}_{o d} \\
& +\frac{\partial \alpha_{w_{i}}}{\partial \phi_{o d}} \dot{\phi}_{o d}+\frac{\partial \alpha_{w_{i}}}{\partial \dot{\phi}_{o d}} \ddot{\phi}_{o d}+\frac{\partial \alpha_{w_{i}}}{\partial q_{i}} \vartheta_{i}+\frac{\partial \alpha_{w_{i}}}{\partial \phi_{i}} w_{i} \\
& +\sum_{j=1, j \neq i}^{N}\left(\frac{\partial \alpha_{w_{i}}}{\partial \phi_{j}} w_{j}+\frac{\partial \alpha_{w_{i}}}{\partial q_{i j}}\left(\vartheta_{i}-\vartheta_{j}\right)\right)
\end{aligned}
$$

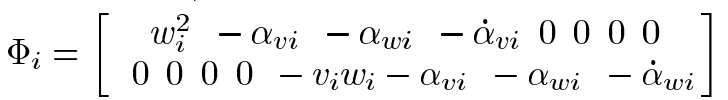

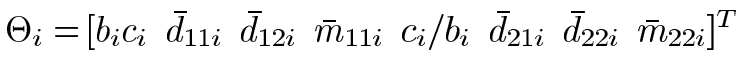

where $\vartheta_{i}=u_{i}+\Delta_{1 i}+\Delta_{2 i}, i=1, \ldots, N$. Again, $\dot{\alpha}_{v_{i}}$ and $\dot{\alpha}_{w_{i}}$ contain only the state and reference trajectory of the robot $i$, and the states of other neighbor robots $j$ if the points $P_{0 j}$ of these robots are in the communication area of the robot $i$, because outside this area $\partial \alpha_{v_{i}} / \partial q_{i j}=0, \partial \alpha_{w_{i}} / \partial q_{i j}=0$, and $\partial \alpha_{w_{i}} / \partial \phi_{j}=0$, thanks to Property 3) of $\beta_{i j}$.

\section{B. Stage II}

In this stage, we design the actual control input vector $\tau_{i}$ and update laws for unknown system parameter vector $\Theta_{i}$ for each robot $i$. To do so, we consider the following function:

$$
\varphi_{I I}=\varphi_{I 2}+\frac{1}{2} \sum_{i=1}^{N}\left(\varpi_{i e}^{T} \bar{M}_{i} \varpi_{i e}+\tilde{\Theta}_{i}^{T} \Gamma_{i}^{-1} \tilde{\Theta}_{i}\right)
$$

where $\tilde{\Theta}_{i}=\Theta_{i}-\hat{\Theta}_{i}$ and $\hat{\Theta}_{i}$ is an estimate of $\Theta_{i}$, and $\Gamma_{i}$ is a symmetric positive definite matrix. Differentiating both sides of (44) along the solutions of (41) and (39) yields

$$
\begin{aligned}
& \dot{\varphi}_{I I}=-k_{0} u_{o d}^{2} \sum_{i=1}^{N} \Omega_{i}^{T} \Psi\left(\Omega_{i}\right)-\sum_{i=1}^{N} k_{i} \phi_{i e}^{2}+\sum_{i=1}^{N} \phi_{i e} w_{i e} \\
&+ \sum_{i=1}^{N}\left(\Omega_{i}^{T}-\phi_{i e} \frac{\partial \alpha_{\phi_{i}}}{\partial q_{i}}\right. \\
&\left.-\sum_{j=1, j \neq i}^{N}\left(\frac{\partial \alpha_{\phi_{i}}}{\partial q_{i j}} \phi_{i e}-\frac{\partial \alpha_{\phi_{j}}}{\partial q_{j i}} \phi_{j e}\right)\right) \Delta_{2 i} \\
&+ \sum_{i=1}^{N}\left(\varpi_{i e}^{T}\left(-\bar{D}_{i} \varpi_{i e}+\Phi_{i} \Theta_{i}+\bar{B}_{i} \tau_{i}\right)\right. \\
&\left.-\tilde{\Theta}_{i}^{T} \Gamma_{i}^{-1} \dot{\hat{\Theta}}_{i}\right)
\end{aligned}
$$

which suggests that we choose (46), shown at the bottom of the page, where $\bar{\Delta}_{2 i}=\left[\cos \left(\phi_{i}\right) \sin \left(\phi_{i}\right)\right]^{T}$, and $L_{i}$ is a symmetric positive definite matrix. Substituting the first equation of (46) into (41) gives

$$
\begin{aligned}
\bar{M}_{i} \dot{\varpi}_{i e}= & -\left(\bar{D}_{i}+L_{i}\right) \varpi_{i e}+\Phi_{i} \tilde{\Theta}_{i} \\
- & {\left[\left(\Omega_{i}^{T}-\phi_{i e} \frac{\partial \alpha_{\phi_{i}}}{\partial q_{i}}\right.\right.} \\
& \left.\left.-\sum_{j=1, j \neq i}^{N}\left(\frac{\partial \alpha_{\phi_{i}}}{\partial q_{i j}} \phi_{i e}-\frac{\partial \alpha_{\phi_{j}}}{\partial q_{j i}} \phi_{j e}\right)\right) \bar{\Delta}_{2 i} \phi_{i e}\right]^{T} .
\end{aligned}
$$

By construction, the control $\tau_{i}$ and the update $\dot{\hat{\Theta}}_{i}$ given in (46) of the robot $i$ contain only the state and reference trajectory of the robot $i$, and the states of other neighbor robots $j$ if these robots are in a circular area, which is centered at point $P_{0 i}$ of the robot

$$
\begin{aligned}
& \tau_{i}=\bar{B}_{i}^{-1}\left(-L_{i} \varpi_{i e}-\Phi_{i} \hat{\Theta}_{i}-\left[\left(\Omega_{i}^{T}-\phi_{i e} \frac{\partial \alpha_{\phi_{i}}}{\partial q_{i}}-\sum_{j=1, j \neq i}^{N}\left(\frac{\partial \alpha_{\phi_{i}}}{\partial q_{i j}} \phi_{i e}-\frac{\partial \alpha_{\phi_{j}}}{\partial q_{j i}} \phi_{j e}\right)\right) \bar{\Delta}_{2 i} \phi_{i e}\right]^{T}\right) \\
& \dot{\hat{\Theta}}_{i}=\Gamma_{i} \Phi_{i}^{T} \varpi_{i e}
\end{aligned}
$$



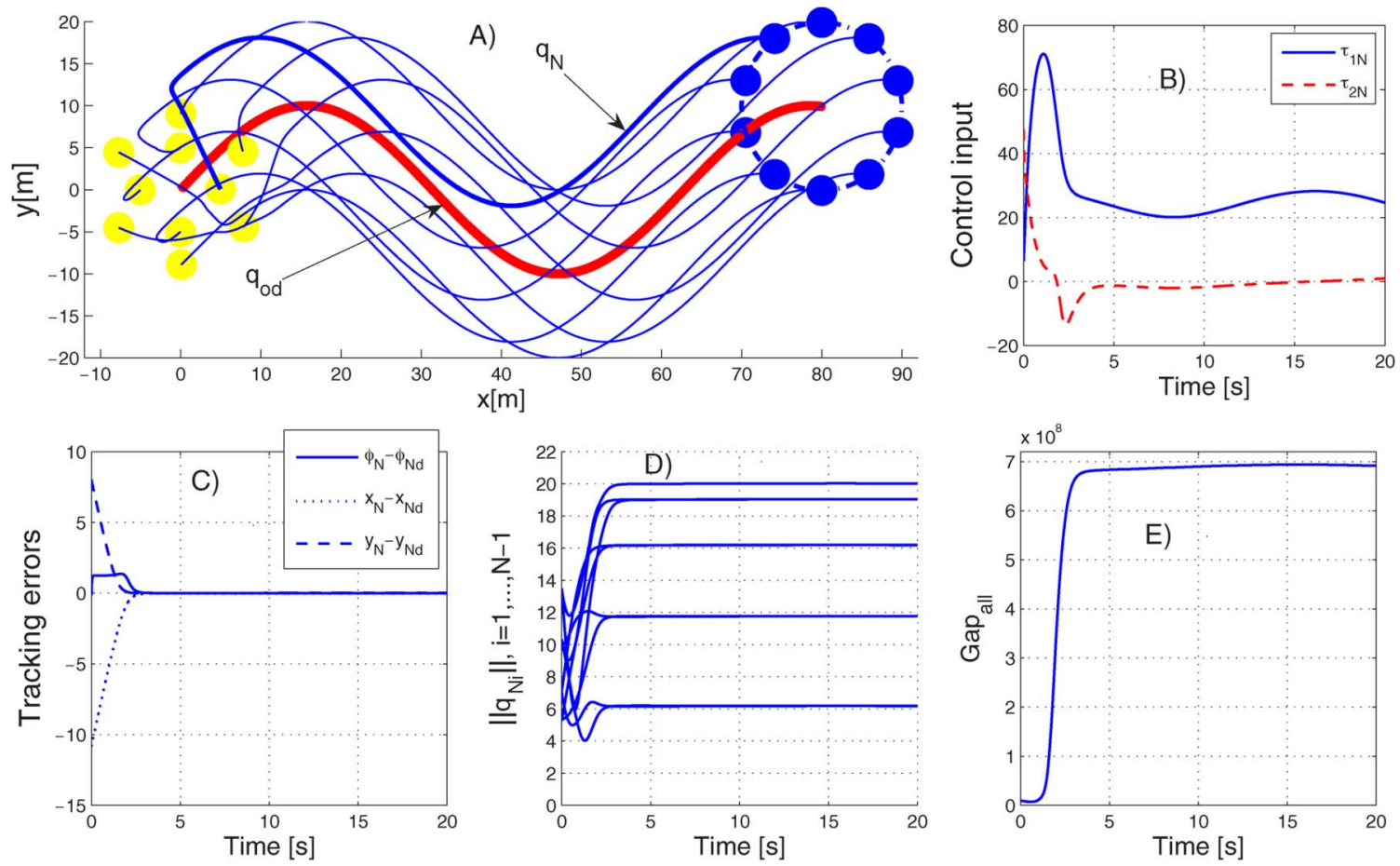

Fig. 4. Simulation results with ten robots.

$i$ and has a radius no greater than $\bar{R}_{i}$. Now, substituting (46) into (45) results in

$\dot{\varphi}_{I I}=-k_{0} u_{o d}^{2} \sum_{i=1}^{N} \Omega_{i}^{T} \Psi\left(\Omega_{i}\right)-\sum_{i=1}^{N} k_{i} \phi_{i e}^{2}-\sum_{i=1}^{N} \varpi_{i e}^{T}\left(\bar{D}_{i}+L_{i}\right) \varpi_{i e}$.

For convenience, we rewrite the closed-loop system consisting of (33), (40), (47), and the second equation of (46) as follows:

$$
\begin{aligned}
\dot{q}_{i}= & -k_{0} u_{o d}^{2} \Psi\left(\Omega_{i}\right)+\dot{q}_{o d}+\Delta_{1 i}+\Delta_{2 i} \\
\dot{\phi}_{i e}= & -k_{i} \phi_{i e}-\frac{\Omega_{i}^{T} \Delta_{1 i}}{\phi_{i e}}-\frac{\partial \alpha_{\phi_{i}}}{\partial q_{i}} \Delta_{2 i} \\
& -\sum_{j=1, j \neq i}^{N} \frac{\partial \alpha_{\phi_{i}}}{\partial q_{i j}}\left(\Delta_{2 i}-\Delta_{2 j}\right)+w_{i e} \\
\bar{M}_{i} \dot{\varpi}_{i e}= & -\left(\bar{D}_{i}+L_{i}\right) \varpi_{i e}+\Phi_{i} \tilde{\Theta}_{i} \\
& -\left[\left(\Omega_{i}^{T}-\phi_{i e} \frac{\partial \alpha_{\phi_{i}}}{\partial q_{i}}\right.\right. \\
& \left.\left.-\sum_{j=1, j \neq i}^{N}\left(\frac{\partial \alpha_{\phi_{i}}}{\partial q_{i j}} \phi_{i e}-\frac{\partial \alpha_{\phi_{j}}}{\partial q_{j i}} \phi_{j e}\right)\right) \bar{\Delta}_{2 i} \phi_{i e}\right]^{T} \\
\dot{\tilde{\Theta}}_{i}= & -\Gamma_{i} \Phi_{i}^{T} \varpi_{i e} .
\end{aligned}
$$

We now state the main result of our brief in the following theorem.

Theorem 1: Under Assumption 1, the control $\tau_{i}$ and the update law $\dot{\hat{\Theta}}_{i}$ given in (46) for the robot $i$ solve the formation control objective. In particular, no collisions between any robots can occur for all $t \geq t_{0} \geq 0$, the closed-loop system (49) is forward complete, and the position and orientation of the robots track their reference trajectories asymptotically in the sense of (11).

Proof: See Appendix B.

\section{Simulations}

Here, we simulate formation tracking control of a group of $N=10$ identical mobile robots to illustrate the effectiveness of the proposed controller. The physical parameters of the robot are taken from [21]: $r_{i}=0.15, a_{i}=0.3, b_{i}=0.5, m_{c i}=$ $30, m_{w i}=1, I_{c i}=15.625, I_{w i}=0.005, I_{m i}=0.0025$, $d_{11 i}=10, d_{22 i}=10, \underline{R}_{i}=1.8$, and $\bar{R}_{i}=5, i=1, \ldots, N$. The robots are initialized as follows: $x_{i}=R_{0} \sin ((i-1) 2 \pi / 6)$, $y_{i}=R_{0} \cos ((i-1) 2 \pi / 6), \omega_{1 i}=0, \omega_{2 i}=0$, where $R_{0}=9$ for $i=1, \ldots, 6$, and $R_{0}=5$ for $i=7, \ldots, N$. The initial values of $\phi_{i}, i=1, \ldots, N$, are taken as random numbers between 0 and $2 \pi$. The initial values of $\hat{\Theta}_{i}$ are taken as half of their true values. The reference trajectories are taken as $q_{o d}=[s 10 \sin (0.1 s)]^{T}$, $\dot{s}=2$ and $l_{i}=10[\sin ((i-1) 2 \pi / N) \cos ((i-1) 2 \pi / N)]^{T}$. This choice of the reference trajectories means that the common reference trajectory $q_{o d}$ forms a sinusoidal trajectory and that the desired formation configuration is a polygon whose vertices uniformly distribute on a circle centered on the common reference trajectory and with a radius 10 . The functions $\beta_{i j},(i, j) \in \mathbb{N}$, $i \neq j$, are taken as in (22) with $p=4, a_{i j}=2 \underline{R}_{i}$, and $b_{i j}=\bar{R}_{i}$. The function $\psi()$ is taken as $\arctan ()$. The control gains and update gains are chosen as $k_{0}=0.1, k_{i}=2, L_{i}=4 I_{2}$, and $\Gamma_{i}=$ $0.2 I_{6}$, where $I_{z}$ is a $z$-dimensional identity matrix. Indeed, the above choice of $k_{0}$ satisfies condition (26). Simulation results are plotted in Fig. 4. It is seen that all robots nicely track their reference trajectories. During the first four seconds, the robots quickly move away from each other to avoid collisions then 
track their desired reference trajectory in Fig. 4(a), where the trajectory of the robot $N$ is plotted in the thick line. For clarity, only the control inputs $\left[\tau_{1 N} \tau_{2 N}\right]^{T}$ of the robot $N$ are plotted in Fig. 4(b). Fig. 4(c) plots the tracking errors $x_{N}-x_{N d}, y_{N}-y_{N d}$, and $\phi_{N}-\phi_{N d}$ of the robot $N$. Indeed, these errors tend to zero asymptotically. The distances between the robot $N$ and other robots are plotted in Fig. 4(d). Clearly, these distances are always larger than $\underline{R}_{N}+\underline{R}_{i}=3.6, i=1, \ldots, N-1$, i.e., there are no collisions between the robot $N$ and all other robots in the group. Moreover, in Fig. 4(e), we plot the product of all gaps between robots: Gap all $_{1}=\prod_{(i, j) \in \mathbb{N}, i \neq j}\left(\left\|q_{i j}\right\|-\left(R_{i}+R_{j}\right)\right)$. It is seen that Gap $_{\text {all }}$ is always larger than zero. This means that $\left(\left\|q_{i j}\right\|-\left(R_{i}+R_{j}\right)>0, \forall(i, j) \in \mathbb{N}, t \geq 0\right.$, i.e., no collisions between any agents occurred. For clarity, we only plot the results for the first $20 \mathrm{~s}$ in Fig. 4(b)-(e).

\section{CONCLUSION}

We have presented a method to design cooperative formation tracking controllers for a group of $N$ unicycle-type mobile robots with limited sensing ranges. The control design was constructed in such a way that the robots asymptotically track their reference trajectories and avoid collisions among them. The novel potential functions with embedded smooth or/and $p$ times differential bump functions are attractive parts of the brief since they do not require the use of switching control theory despite the robot limited sensing ranges. These functions can certainly be modified to solve other cooperative control problems such as flocking and consensus of mobile agents. In addition, the method of constructing the virtual controls $\alpha_{\phi_{i}}$ and $\alpha_{v_{i}}$ is also attractive, since they are derived directly from the position tracking errors instead of transforming the robot kinematics to a body frame as often done in the literature. Future work is an extension of the proposed techniques in this brief and those for single underactuated ocean vessels in [28] and [29] to achieve a desired formation for a group of underactuated vessels.

\section{APPENDIX A}

\section{PROOF OF LEMMA 1}

We need to verify that the function $h(x, a, b)$ given in (13) satisfies all properties defined in (12). Property 1) holds because, by (14), for all $0 \leq x \leq a$, we have $\int_{a}^{x} f(\tau-a) f(b-\tau) d \tau=0$. Property 2$)$ holds since, by (14), we have $\int_{a}^{x} f(\tau-a) f(b-\tau) d \tau=\int_{a}^{b} f(\tau-a) f(b-\tau) d \tau$ for all $x \geq b$. Property 3 ) holds because it is not hard to show that $0<\left(\int_{a}^{x} f(\tau-a) f(b-\tau) d \tau / \int_{a}^{b} f(\tau-a) f(b-\tau) d \tau\right)<1$ for all $a<x<b$. To prove Property 4 ), we just need to show that $f(y)$ is $p-1$ times differentiable. We first note that $f(y)$ is smooth except at $y=0$. Hence, we only need to verify that $f^{(k)}(0)=\left.\left(\partial^{k} f(y) / \partial y^{k}\right)\right|_{y=0}=0$ for any positive integer $k<p$. Clearly, $\lim _{y \rightarrow 0^{-}} f^{(k)}(y)=0$ since $f(y)=0, \forall y \leq 0$. On the other hand, since $f(y)=y^{p}$, $y>0$ we have $\lim _{y \rightarrow 0^{+}} f^{(k)}(y)=0$. Since both left- and right-hand limits are equal to 0 , we have $f^{(k)}(0)=0$. Hence, Property 4) holds. Now we turn to the case where $f(y)=y^{p}$ in (14) is replaced by $f(y)=e^{-1 / y}$. Properties 1)-3) can be proven without difficulty. We focus on proof of Property $4^{\prime}$ ). We first note that $f^{(k)}(y)=\partial^{k} f(y) / \partial y^{k}=Q_{k}(1 / y) e^{-(1 / y) \text {, }}$ where $Q_{k}(1 / y)$ is a polynomial function of $1 / y$, and $k$ is any positive integer. We will prove Property $4^{\prime}$ ) by induction. First of all, we check that $f^{(1)}(0)=0$. It is clear that $\lim _{y \rightarrow 0^{-}}((f(y)-f(0)) / y-0)=\lim _{y \rightarrow 0^{-}}(0 / y)=0$. On the other hand, $\lim _{y \rightarrow 0^{+}}((f(y)-f(0)) / y-0)=$ $\lim _{y \rightarrow 0^{+}}\left(e^{-(1 / y)} / y\right)=\lim _{\xi \rightarrow \infty}\left(1 / e^{\xi}\right)=0$ where $\xi=1 / y$ and we have used l'Hopital's rule. Since both left- and right-hand limits are equal to 0 , we have $f^{(1)}(0)=0$. Now assume that $f^{(k)}(0)=0$. We now compute $f^{(k+1)}(0)$. The left-hand limit is equal to 0 as above. The right hand $\operatorname{limit}_{\text {is }} \lim _{y \rightarrow 0^{+}}\left(\left(f^{(k)}(y)-\right.\right.$ $\left.\left.f^{(k)}(0)\right) /(y-0)\right)=\lim _{y \rightarrow 0^{+}}\left(\left(f^{(k)}(y)-0\right) /(y-0)\right)=$ $\lim _{y \rightarrow 0^{+}}(1 / y) Q_{k}(1 / y) e^{-(1 / y)}=\lim _{y \rightarrow 0^{+}} \tilde{Q}_{k}(1 / y) e^{-(1 / y)}=$ $\lim _{\xi \rightarrow \infty}\left(\tilde{Q}_{k}(\xi) / e^{\xi}\right)=0$ (by l'Hopital's rule), where $\xi=1 / y$ and $\tilde{Q}_{k}(\xi)=\xi Q_{k}(\xi)$ is another polynomial of $1 / y$. Since both left- and right-hand limits are equal to 0 , we have $f^{(k+1)}(0)=0$, which completes the proof of Property $\left.4^{\prime}\right)$.

\section{APPENDIX B \\ PROOF OF THEOREM 1}

We first prove that no collisions between the robots can occur, the closed-loop system (49) is forward complete and that the robots asymptotically approach their target points or some critical points. We then investigate stability of the closed-loop system (49) at these points and show that the position and orientation of the robots asymptotically track their reference trajectories.

Proof of No Collisions and Complete Forwardness of Closed Loop System: From (48) and properties of the function $\psi$ [see (26)], we have $\dot{\varphi}_{I I} \leq 0$, which implies that $\varphi_{I I}(t) \leq \varphi_{I I}\left(t_{0}\right)$, $\forall t \geq t_{0}$. With definition of the function $\varphi_{I I}$ in (44) and its components in (36), (18), (19), and (20), we have

$$
\begin{aligned}
\sum_{i=1}^{N}\left[\gamma_{i}(t)\right. & +\frac{1}{2} \sum_{j \in \mathbb{N}_{i}} \beta_{i j}(t)+\frac{1}{2} \phi_{i e}(t)+\frac{1}{2} \varpi_{i e}^{T}(t) \bar{M}_{i} \varpi_{i e}(t) \\
+ & \left.\frac{1}{2}\left(\Theta_{i}-\hat{\Theta}_{i}(t)\right)^{T} \Gamma_{i}^{-1}\left(\Theta_{i}-\hat{\Theta}_{i}(t)\right)\right] \\
\leq \sum_{i=1}^{N} & {\left[\gamma_{i}\left(t_{0}\right)+\frac{1}{2} \sum_{j \in \mathbb{N}_{i}} \beta_{i j}\left(t_{0}\right)+\frac{1}{2} \phi_{i e}\left(t_{0}\right)\right.} \\
& +\frac{1}{2} \varpi_{i e}^{T}\left(t_{0}\right) \bar{M}_{i} \varpi_{i e}\left(t_{0}\right) \\
& \left.+\frac{1}{2}\left(\Theta_{i}-\hat{\Theta}_{i}\left(t_{0}\right)\right)^{T} \Gamma_{i}^{-1}\left(\Theta_{i}-\hat{\Theta}_{i}\left(t_{0}\right)\right)\right]
\end{aligned}
$$

for all $t \geq t_{0} \geq 0$. From the condition specified in item 4) of Assumption 1, Property 5) of $\beta_{i j}$, and the definition of $\phi_{i e}$, $\varpi_{i e}$, the right-hand side of (50) is bounded by a positive constant depending on the initial conditions. The boundedness of the right-hand side of (50) implies that the left-hand side of (50) must be also bounded. As a result, $\beta_{i j}(t)$ must be smaller than some positive constant depending on the initial conditions for all $t \geq t_{0} \geq 0$. From properties of $\beta_{i j}$ [see (21)], $\left\|q_{i j}(t)\right\|-$ $\left(\underline{R}_{i}+\underline{R}_{j}\right)$ must be larger than some positive constant depending on the initial conditions denoted by $\epsilon_{3}$, i.e., there are no collisions for all $t \geq t_{0} \geq 0$. Boundedness of the left-hand side of (50) also implies that of $\left(q_{i}(t)-q_{i d}(t)\right), \phi_{i e}(t), \varpi_{i e}(t)$, and 
$\hat{\Theta}_{i}(t)$ for all $t \geq t_{0} \geq 0$. This in turn implies by construction that $x_{i}(t), y_{i}(t), \phi_{i}(t), v_{i}(t)$, and $w_{i}(t)$ do not escape to infinity in finite time. Therefore, the closed-loop system (49) is forward-complete.

Equilibrium Points: Since we have already proved that there are no collisions between any robots, an application of [26, Theorem 8.4 ] to (48) yields

$$
\begin{aligned}
\lim _{t \rightarrow \infty}\left(k_{0} u_{o d}^{2}(t) \sum_{i=1}^{N}\right. & \Omega_{i}^{T}(t) \Psi\left(\Omega_{i}(t)\right)+\sum_{i=1}^{N} k_{i} \phi_{i e}^{2}(t) \\
& \left.+\sum_{i=1}^{N} \varpi_{i e}^{T}(t)\left(\bar{D}_{i}+L_{i}\right) \varpi_{i e}(t)\right)=0 .
\end{aligned}
$$

By noting that $\lim _{t \rightarrow \infty} u_{o d}^{2}(t) \neq 0$ as specified in item 5) of Assumption 1, the limit equation (51) implies that

$$
\lim _{t \rightarrow \infty} \Omega_{i}(t)=0, \quad \lim _{t \rightarrow \infty} \phi_{i e}(t)=0, \lim _{t \rightarrow \infty} \varpi_{i e}(t)=0 .
$$

By construction, $\lim _{t \rightarrow \infty} \Omega_{i}(t)=0$ and $\lim _{t \rightarrow \infty} \phi_{i e}(t)=0$ imply that $\lim _{t \rightarrow \infty}\left(\phi_{i}(t)-\phi_{\text {od }}(t)\right)=0$. Moreover, from the definition of $\Omega_{i}$ in (24), $\lim _{t \rightarrow \infty} \Omega_{i}(t)=0$ means

$$
\lim _{t \rightarrow \infty}\left(q_{i}(t)-q_{i d}(t)+\sum_{j \in \mathbb{N}_{i}} \beta_{i j}^{\prime} q_{i j}(t)\right)=0 .
$$

The limit equation (52) implies that $q(t)=$ $\left[q_{1}^{T}(t) q_{2}^{T}(t), \ldots, q_{N}^{T}(t)\right]^{T} \quad$ can tend to $q_{d}=$ $\left[q_{1 d}^{T} q_{2 d}^{T}, \ldots, q_{N d}^{T}\right]^{T}$ since $\beta_{i j}^{\prime} \|_{\left\|q_{i j}\right\|=\left\|q_{i j d}\right\|}=0$ (Property 1) of $\beta_{i j}$ ), or some vector denoted by $q_{c}=\left[q_{1 c}^{T} q_{2 c}^{T}, \ldots, q_{N c}^{T}\right]^{T}$ as the time goes to infinity, i.e., the equilibrium points can be $q_{d}$ or $q_{c}$. It is noted that some elements of $q_{c}$ can be equal to that of $q_{d}$. However, for simplicity, we abuse the notation, i.e., we still denote that vector as $q_{c}$. Indeed, the vector $q_{c}$ is such that

$$
\left.\Omega_{i}\right|_{q=q_{c}}=\left.\left[q_{i}-q_{i d}+\sum_{j \in \mathbb{N}_{i}} \beta_{i j}^{\prime} q_{i j}\right]\right|_{q=q_{c}}=0 \quad \forall i=1, \ldots, N .
$$

To investigate properties of the equilibrium points, $q_{d}$ and $q_{c}$, we consider the first equation of the closed-loop system (49), i.e.,

$$
\dot{q}_{i}=-k_{0} u_{o d}^{2} \Psi\left(\Omega_{i}\right)+\dot{q}_{o d}+\Delta_{1 i}+\Delta_{2 i} .
$$

Since we have already proved that the closed-loop system (49) is forward complete, and $\lim _{t \rightarrow \infty} \phi_{i e}(t)=0$ and $\lim _{t \rightarrow \infty} \varpi_{i e}(t)=0$ imply from the expressions of $\Delta_{1 i}$ and $\Delta_{2 i}$ [see (17)] that $\lim _{t \rightarrow \infty}\left(\Delta_{1 i}(t)+\Delta_{2 i}(t)\right)=0$, we treat $\Delta_{i}(t)=\Delta_{1 i}(t)+\Delta_{2 i}(t)$ as an input to (54) instead of a state. Moreover, we have already proved that the trajectory $q$ can approach either the set of desired equilibrium points denoted by $q_{d}$ or the set of undesired equilibrium points denoted by $q_{c}$ "almost globally." The term "almost globally" refers to the fact that the agents start from a set that includes both condition (7) and that does not coincide at any point with the set of the undesired saddle point $q_{c}$. Therefore, we now need to prove that $q_{d}$ is locally asymptotically stable and that $q_{c}$ is locally unstable. Once this is proved, we can conclude that the trajectory $q$ approaches $q_{d}$ from almost everywhere except for from the set denoted by the condition (7) and the set denoted by $q_{c}$, which is unstable.
Properties of Equilibrium Points: The system (54) can be written in a vector form as

$$
\dot{q}=-k_{0} u_{o d}^{2} \Psi_{q}\left(q, q_{d}\right)+\operatorname{vec}\left(\dot{q}_{o d}\right)+\operatorname{vec}\left(\Delta_{i}\right)
$$

where $\Psi_{q}\left(q, q_{d}\right)=\left[\Psi^{T}\left(\Omega_{1}\right), \ldots, \Psi^{T}\left(\Omega_{i}\right), \ldots, \Psi^{T}\left(\Omega_{N}\right)\right]^{T}$, $\operatorname{vec}\left(\dot{q}_{o d}\right)=\left[\dot{q}_{o d}^{T}, \ldots, \dot{q}_{o d}^{T}, \ldots, \dot{q}_{o d}^{T}\right]^{T}$, and $\operatorname{vec}\left(\Delta_{i}\right)=$ $\left[\Delta_{1}^{T}, \ldots, \Delta_{i}^{T}, \ldots, \Delta_{N}^{T}\right]^{T}$. Therefore, near an equilibrium point $q_{o}$, which can be either $q_{d}$ or $q_{c}$, we have

$\dot{q}=-k_{0} u_{o d}^{2} \partial \Psi_{q}\left(q, q_{d}\right) /\left.\partial q\right|_{q=q_{o}}\left(q-q_{o}\right)+\operatorname{vec}\left(\dot{q}_{o d}\right)+\operatorname{vec}\left(\Delta_{i}\right)$

where the $\left(i^{t h}, j^{t h}\right)$ element of the matrix $\partial \Psi_{q}\left(q, q_{d}\right) / \partial q$ is $\bigwedge_{i j}=\left(\partial \Psi\left(\Omega_{i}\right) / \partial \Omega_{i}\right)\left(\partial \Omega_{i} / \partial q_{j}\right),(i, j) \in \mathbb{N}$, where $\mathbb{N}$ is the set of all agents. A simple calculation shows that

$$
\begin{aligned}
& \frac{\partial \Omega_{i}}{\partial q_{i}}=\left(1+\sum_{i \in \mathbb{N}_{i}} \beta_{i j}^{\prime}\right) I_{n}+\sum_{j \in \mathbb{N}_{i}} \beta_{i j}^{\prime \prime} q_{i j} q_{i j}^{T} \\
& \frac{\partial \Omega_{i}}{\partial q_{j}}=-\beta_{i j}^{\prime} I_{n}-\beta_{i j}^{\prime \prime} q_{i j} q_{i j}^{T}
\end{aligned}
$$

for all $i=1, \ldots, N, j \in \mathbb{N}_{i}, j \neq i$, where $I_{n}$ denotes the identity matrix of size $n$. Let $\mathbb{N}^{*}$ be the set of the agents such that, if the agents $i$ and $j$ belong to the set $\mathbb{N}^{*}$, then $\left\|q_{i j}\right\|<b_{i j}$. Next, we will show that $q_{d}$ is asymptotically stable and that $q_{c}$ is unstable.

Proof of $q_{d}$ Being Asymptotic Stable: Using properties of $\beta_{i j}$ and $\psi$ listed in (21) and (26), we have from (57) that for all $i=1, \ldots, N, i \neq j$

$$
\begin{aligned}
\left.\frac{\partial \Psi\left(\Omega_{i}\right)}{\partial \Omega_{i}}\right|_{q=q_{d}} & =I_{n}, \quad \beta_{i j d}^{\prime}=0 \\
\left.\frac{\partial \Omega_{i}}{\partial q_{i}}\right|_{q=q_{d}} & =I_{n}+\sum_{j \in \mathbb{N}_{i}} \beta_{i j d}^{\prime \prime} q_{i j d} q_{i j d}^{T} \\
\left.\frac{\partial \Omega_{i}}{\partial q_{j}}\right|_{q=q_{d}} & =-\beta_{i j d}^{\prime \prime} q_{i j d} q_{i j d}^{T}
\end{aligned}
$$

where $\beta_{i j d}^{\prime}=\left.\beta_{i j}^{\prime}\right|_{q_{i j}=q_{i j d}}$ and $\beta_{i j d}^{\prime \prime}=\left.\beta_{i j}^{\prime \prime}\right|_{q_{i j}=q_{i j d}}$, with $q_{i j d}=$ $q_{i d}-q_{j d}$. We consider the function

$$
V_{d}=\sqrt{1+\left\|q-q_{d}\right\|^{2}}-1
$$

whose derivative along the solutions of (56) with $q_{o}$ replaced by $q_{d}$, using (58), and noting that $\dot{q}_{o d}=\dot{q}_{i d}$ satisfies

$$
\begin{aligned}
& \dot{V}_{d}= \frac{1}{\sqrt{1+\left\|q-q_{d}\right\|^{2}}} \\
& \times\left(-k_{0} u_{o d}^{2} \sum_{i=1}^{N}\left\|q_{i}-q_{i d}\right\|^{2}\right. \\
&-k_{0} u_{o d}^{2} \sum_{(i, j) \in \mathbb{N}, i \neq j} \beta_{i j d}^{\prime \prime}\left(q_{i j d}^{T}\left(q_{i j}-q_{i j d}\right)\right)^{2} \\
&\left.+\sum_{i=1}^{N}\left(q_{i}-q_{i d}\right)^{T} \Delta_{i}\right) \\
& \leq-\frac{k_{0} u_{o d}^{2}}{\sqrt{1+\left\|q-q_{d}\right\|^{2}}} \sum_{i=1}^{N}\left\|q_{i}-q_{i d}\right\|^{2}+\sum_{i=1}^{N}\left\|\Delta_{i}\right\|
\end{aligned}
$$

since $\beta_{i j d}^{\prime \prime} \geq 0$ [see Property 1) in (21)]. The last inequality of (60) implies that $q_{d}$ is asymptotically stable because 
$\lim _{t \rightarrow \infty} u_{o d}^{2}(t) \neq 0$, and we have already proved that $\lim _{t \rightarrow \infty} \Delta_{i}(t)=0$.

Proof of $q_{c}$ Being Unstable: Again, using properties of $\beta_{i j}$ and $\psi$ in (21) and (26), we have from (57) that

$$
\begin{aligned}
\left.\frac{\partial \Psi\left(\Omega_{i}\right)}{\partial \Omega_{i}}\right|_{q=q_{c}} & =I_{n} \\
\left.\frac{\partial \Omega_{i}}{\partial q_{i}}\right|_{q=q_{c}} & =\left(1+\sum_{j \in \mathbb{N}_{i}} \beta_{i j c}^{\prime}\right) I_{n}+\sum_{j \in \mathbb{N}_{i}} \beta_{i j c}^{\prime \prime} q_{i j c} q_{i j c}^{T} \\
\left.\frac{\partial \Omega_{i}}{\partial q_{j}}\right|_{q=q_{c}} & =-\beta_{i j c}^{\prime}-\beta_{i j c}^{\prime \prime} q_{i j c} q_{i j c}^{T}
\end{aligned}
$$

for all $i=1, \ldots, N, i \neq j$, where $q_{i j c}=q_{i c}-q_{j c}, \beta_{i j c}^{\prime}=$ $\left.\beta_{i j}^{\prime}\right|_{q_{i j}=q_{i j c}}$, and $\beta_{i j c}^{\prime \prime}=\left.\beta_{i j}^{\prime \prime}\right|_{q_{i j}=q_{i j c}}$. Since the related collision avoidance functions $\beta_{i}$ [see (20)] are specified in terms of relative distances between agents and it is extremely difficult to obtain $q_{c}$ explicitly by solving (53), it is very difficult to use the Lyapunov function candidate $V_{c}=0.5\left\|q-q_{c}\right\|$ to investigate stability of (56) at $q_{c}$. Therefore, we consider the Lyapunov function candidate

$$
\bar{V}_{c}=\sqrt{1+\left\|\bar{q}-\overline{q_{c}}\right\|^{2}}-1
$$

where $\bar{q}=\left[q_{12}^{T}, q_{13}^{T}, \ldots, q_{1 N}^{T}, q_{23}^{T}, \ldots, q_{2 N}^{T}, \ldots, q_{N-1, N}^{T}\right]^{T}$ and $\overline{q_{c}}=\left[q_{12 c}^{T}, q_{13 c}^{T}, \ldots, q_{1 N c}^{T}, q_{23 c}^{T}, \ldots, q_{2 N c}^{T}, \ldots, q_{N-1, N c}^{T}\right]^{T}$. Differentiating both sides of (62) along the solution of (56) with $q_{o}$ replaced by $q_{c}$ gives

$$
\begin{aligned}
\dot{\bar{V}}_{c}= & -\frac{k_{0} u_{o d}^{2}}{\sqrt{1+\left\|\bar{q}-\bar{q}_{c}\right\|^{2}}} \sum_{(i, j) \in \mathbb{N}_{\mathbb{N}^{*}}}\left\|q_{i j}-q_{i j c}\right\|^{2} \\
& -\frac{k_{0} u_{o d}^{2}}{\sqrt{1+\left\|\bar{q}-\bar{q}_{c}\right\|^{2}}} \sum_{(i, j) \in \mathbb{N}^{*}}\left(1+N \beta_{i j c}^{\prime}\right)\left\|q_{i j}-q_{i j c}\right\|^{2} \\
& -\frac{k_{0} u_{o d}^{2} N}{\sqrt{1+\left\|\bar{q}-\overline{q_{c}}\right\|^{2}}} \sum_{(i, j) \in \mathbb{N}^{*}} \beta_{i j c}^{\prime \prime}\left(q_{i j c}^{T}\left(q_{i j}-q_{i j c}\right)\right)^{2} \\
& +\frac{1}{\sqrt{1+\left\|\bar{q}-\overline{q_{c}}\right\|^{2}}} \sum_{(i, j) \in \mathbb{N}}\left(q_{i j}-q_{i j c}\right)^{T}\left(\Delta_{i}-\Delta_{j}\right)
\end{aligned}
$$

where $i \neq j$ and (61) has been used. To investigate stability properties of $\overline{q_{c}}$ based on (63), we will use (53). Define $\Omega_{i j c}=$ $\Omega_{i c}-\Omega_{j c}, \forall(i, j) \in\{1, \ldots, N\}, i \neq j$ where $\Omega_{i c}=\left.\Omega_{i}\right|_{q=q_{c}}=$ $0[$ see $(53)]$. Therefore, $\Omega_{i j c}=0$. Hence, $\sum_{(i, j) \in \mathbb{N}^{*}} q_{i j c}^{T} \Omega_{i j c}=$ $0, i \neq j$, which, by using (53), is expanded to

$$
\begin{aligned}
& \sum_{(i, j) \in \mathbb{N}^{*}}\left(q_{i j c}^{T}\left(q_{i j c}-q_{i j d}\right)+N \beta_{i j c}^{\prime} q_{i j c}^{T} q_{i j c}\right)=0 \\
& \Rightarrow \sum_{(i, j) \in \mathbb{N}^{*}}\left(1+N \beta_{i j c}^{\prime}\right) q_{i j c}^{T} q_{i j c}=\sum_{(i, j) \in \mathbb{N}^{*}} q_{i j c}^{T} q_{i j d}
\end{aligned}
$$

where $i \neq j$. The sum $\sum_{(i, j) \in \mathbb{N}^{*}} q_{i j c}^{T} q_{i j d}$ is strictly negative since, at the point, say $F$, where $q_{i j}=q_{i j d}, \forall(i, j) \in \mathbb{N}^{*}, i \neq j$, all attractive and repulsive forces are equal to zero, while at the point, say $C$, where $q_{i j}=q_{i j c} \forall(i, j) \in \mathbb{N}^{*}, i \neq j$, the sum of attractive and repulsive forces are equal to zero (but attractive and repulsive forces are nonzero). Therefore, the point, say $O$, where $q_{i j}=0, \forall(i, j) \in \mathbb{N}^{*}, i \neq j$, must locate between the points $F$ and $C$ for all $(i, j) \in \mathbb{N}^{*}, i \neq j$, i.e., there exists a strictly positive constant $b$ such that $\sum_{(i, j) \in \mathbb{N}^{*}} q_{i j c}^{T} q_{i j d}<-b$, which is substituted into (64) to yield

$$
\sum_{(i, j) \in \mathbb{N}^{*}}\left(1+N \beta_{i j c}^{\prime}\right) q_{i j c}^{T} q_{i j c}<-b, \quad i \neq j .
$$

Since $q_{i j c}^{T} q_{i j c}>0, \forall(i, j) \in \mathbb{N}^{*}, i \neq j$, there exists a nonempty set $\mathbb{N}^{* *} \subset \mathbb{N}^{*}$ such that, for all $(i, j) \in \mathbb{N}^{* *}, i \neq j,\left(1+N \beta_{i j c}^{\prime}\right)$ is strictly negative, i.e., there exists a strictly positive constant $b^{* *}$ such that $\left(1+N \beta_{i j c}^{\prime}\right)<-b^{* *}, \forall(i, j) \in \mathbb{N}^{* *}, i \neq j$. We now write (63) as

$$
\begin{aligned}
\dot{\bar{V}}_{c}= & -\frac{k_{0} u_{o d}^{2}}{\sqrt{1+\left\|\bar{q}-\bar{q}_{c}\right\|^{2}}} \\
& \times\left[\sum_{(i, j) \in \mathbb{N} \backslash \mathbb{N}^{*}}\left\|q_{i j}-q_{i j c}\right\|^{2}\right. \\
& +\sum_{(i, j) \in \mathbb{N}^{*} \backslash \mathbb{N}^{* *}}\left(1+N \beta_{i j c}^{\prime}\right)\left\|q_{i j}-q_{i j c}\right\|^{2} \\
& +\frac{k_{0} u_{o d}^{2}}{\sqrt{1+\| \bar{q}-\overline{q_{c} \|^{2}}}} \beta_{(i, j) \in \mathbb{N}^{* *}}\left(1+N \beta_{i j c}^{\prime}\right)\left\|q_{i j}-q_{i j c}\right\|^{2} \\
+ & \left.\left.\frac{1}{\sqrt{1+\| \bar{q}-\overline{q_{c} \|^{2}}}} \sum_{(i, j) \in \mathbb{N}^{*}}\left(q_{i j}-q_{i j c}\right)\right)^{2}\right]
\end{aligned}
$$

where $i \neq j$. We now define a subspace such that $q_{i j}-q_{i j c}=0$, $\forall(i, j) \in \mathbb{N} \backslash \mathbb{N}^{* *}$ and $q_{i j c}^{T}\left(q_{i j}-q_{i j c}\right)=0, \forall(i, j) \in \mathbb{N}^{*}, i \neq j$. In this subspace, we have

$$
\begin{aligned}
\bar{V}_{c}= & \sqrt{1+\sum_{(i, j) \in \mathbb{N}^{* *}}\left\|q_{i j}-q_{i j c}\right\|^{2}}-1 \\
\dot{\bar{V}}_{c}= & -\frac{k_{0} u_{o d}^{2} \sum_{(i, j) \in \mathbb{N}^{* *}}\left(1+N \beta_{i j c}^{\prime}\right)\left\|q_{i j}-q_{i j c}\right\|^{2}}{\sqrt{1+\sum_{(i, j) \in \mathbb{N}^{* *}\left\|q_{i j}-q_{i j c}\right\|^{2}}}} \\
& +\frac{\sum_{(i, j) \in \mathbb{N}^{* *}}\left(q_{i j}-q_{i j c}\right)^{T}\left(\Delta_{i}-\Delta_{j}\right)}{\sqrt{1+\sum_{(i, j) \in \mathbb{N}^{* *}\left\|q_{i j}-q_{i j c}\right\|^{2}}}}
\end{aligned}
$$

Using $\left(1+N \beta_{i j c}^{\prime}\right)<-b^{* *}, \forall(i, j) \in \mathbb{N}^{* *}, i \neq j$, we can write (67) as

$$
\begin{aligned}
& \bar{V}_{c}=\sqrt{1+\sum_{(i, j) \in \mathbb{N}^{* *}}\left\|q_{i j}-q_{i j c}\right\|^{2}}-1, \\
& \dot{\bar{V}}_{c} \geq b^{* *} k_{0} u_{o d}^{2} \bar{V}_{c}-\sum_{(i, j) \in \mathbb{N}^{* *}}\left\|\Delta_{i}-\Delta_{j}\right\| .
\end{aligned}
$$

Now assume that $q_{c}$ is a stable equilibrium point, i.e., $\lim _{t \rightarrow \infty}\left\|q_{i}(t)-q_{i c}\right\|=d_{i}, \forall i \in \mathbb{N}$, where $d_{i}$ is a nonnegative constant. Noting that $\mathbb{N}^{* *} \subset \mathbb{N}$, we have $\lim _{t \rightarrow \infty}\left\|q_{i}(t)-q_{i c}\right\|=d_{i}, \forall i \in \mathbb{N}^{* *}$, which implies that $\lim _{t \rightarrow \infty} \sum_{(i, j) \in \mathbb{N}^{* *}}\left\|q_{i j}(t)-q_{i j c}\right\|=d^{* *}$, 
$\forall(i, j) \in \mathbb{N}^{* *}, i \neq j$, where $d^{* *}$ is a nonnegative constant, since $q_{i j}=q_{i}-q_{j}$ and $q_{i j c}=q_{i c}-q_{j c}$. We now consider two cases: $\sum_{(i, j) \in \mathbb{N}^{* *}}\left\|q_{i j}\left(t_{0}\right)-q_{i j c}\right\| \neq 0$ and $\sum_{(i, j) \in \mathbb{N}^{* *}}\left\|q_{i j}\left(t_{0}\right)-q_{i j c}\right\|=0$.

Case I: $\sum_{(i, j) \in \mathbb{N}^{* *}}\left\|q_{i j}\left(t_{0}\right)-q_{i j c}\right\| \neq 0$ : Since $\lim _{t \rightarrow \infty} u_{o d}^{2}(t) \stackrel{\neq}{\neq} 0$ (Assumption 1) and we have already shown that $\lim _{t \rightarrow \infty} \Delta_{i}(t)=0, \forall i \in \mathbb{N}, \bar{V}_{c}$ in (68) is divergent. Therefore, $\sum_{(i, j) \in \mathbb{N}^{* *}}\left\|q_{i j}(t)-q_{i j c}\right\|$ cannot tend to a constant but must be divergent. This contradicts $\lim _{t \rightarrow \infty} \sum_{(i, j) \in \mathbb{N}^{* *}}\left\|q_{i j}(t)-q_{i j c}\right\|=d^{* *}$, i.e., $q_{c}$ cannot be a set of stable equilibrium points but must be a set of an unstable ones in this case.

Case I: $\sum_{(i, j) \in \mathbb{N}^{* *}}\left\|q_{i j}\left(t_{0}\right)-q_{i j c}\right\|=0$ : There would be no contradiction. However, this case is never observed in practice since the ever-present physical noise would cause $\sum_{(i, j) \in \mathbb{N}^{* *}}\left\|q_{i j}\left(t^{*}\right)-q_{i j c}\right\|$ to be different from zero at the time $t^{*}>t_{0}$. We now need to show that, once the sum $\sum_{(i, j) \in \mathbb{N}^{* *}}\left\|q_{i j}\left(t^{*}\right)-q_{i j c}\right\|$ is different from zero, this sum will not come back zero again for all $t \geq t^{*}$, i.e., the set of undesired equilibrium points $q_{c}$ is not attractive. To do so, consider (68) with the initial time $t^{*}$ instead of $t_{0}$, i.e.,

$$
\begin{aligned}
& \bar{V}_{c}(t)=\sqrt{1+\sum_{(i, j) \in \mathbb{N}^{* *}}\left\|q_{i j}(t)-q_{i j c}\right\|^{2}}-1 \\
& \dot{\bar{V}}_{c}(t) \geq b^{* *} k_{0} u_{o d}^{2} \bar{V}_{c}(t)-\sum_{(i, j) \in \mathbb{N}^{* *}}\left\|\Delta_{i}(t)-\Delta_{j}(t)\right\|
\end{aligned}
$$

for $t \geq t^{*}$ and $\sum_{(i, j) \in \mathbb{N}^{* *}}\left\|q_{i j}\left(t^{*}\right)-q_{i j c}\right\| \geq \delta^{*}$, where $\delta^{*}$ is a positive constant. Since $\lim _{t \rightarrow \infty} u_{o d}^{2}(t) \neq 0$ (Assumption 1) and we have already shown that $\lim _{t \rightarrow \infty} \Delta_{i}(t)=0, \forall i \in \mathbb{N}, \bar{V}_{c}$ in (69) is divergent for $t \geq t^{*}$. Therefore, $\sum_{(i, j) \in \mathbb{N}^{* *}} \| q_{i j}(t)-$ $q_{i j c} \|$, for $t \geq t^{*}$, cannot tend to a constant but must be divergent. This contradicts $\lim _{t \rightarrow \infty} \sum_{(i, j) \in \mathbb{N}^{* *}}\left\|q_{i j}(t)-q_{i j c}\right\|=d^{* *}$, i.e., $q_{c}$ must also be a set of unstable ones point in this case. The proof of Theorem 1 is completed.

\section{ACKNOWLEDGMENT}

The author would like to thank the reviewers for their helpful comments which helped him improve the brief significantly.

\section{REFERENCES}

[1] A. K. Das, R. Fierro, V. Kumar, J. P. Ostrowski, J. Spletzer, and C. J. Taylor, "A vision based formation control framework," IEEE Trans. Robot. Autom., vol. 18, no. 5, pp. 813-825, Oct. 2002.

[2] N. E. Leonard and E. Fiorelli, "Virtual leaders, artificial potentials and coordinated control of groups," in Proc. 40th IEEE Conf. Decision Control, Orlando, FL, 2001, pp. 2968-2973.

[3] R. T. Jonathan, R. W. Beard, and B. J. Young, "A decentralized approach to formation maneuvers," IEEE Trans. Robot. Autom., vol. 19, no. 6, pp. 933-941, Dec. 2003.

[4] T. Balch and R. C. Arkin, "Behavior-based formation control for multirobot teams," IEEE Trans. Robot. Autom., vol. 14, no. 6, pp. 926-939, Dec. 1998.

[5] M. A. Lewis and K.-H. Tan, "High precision formation control of mobile robots using virtual structures," Auton. Robots, vol. 4, no. 4, pp. 387-403, 1997.
[6] R. Skjetne, S. Moi, and T. I. Fossen, "Nonlinear formation control of marine craft," in Proc. 41st IEEE Conf. Decision Control, Las Vegas, NV, 2002, pp. 1699-1704.

[7] H. G. Tanner and A. Kumar, "Towards decentralization of multi-robot navigation functions," in Proc. IEEE Int. Conf. Robot. Autom., Barcelona, Spain, 2005, pp. 4132-4137.

[8] P. Ogren, M. Egerstedt, and X. Hu, "A control Lyapunov function approach to multi-agent coordination," IEEE Trans. Robot. Autom., vol. 18, no. 5, pp. 847-851, Oct. 2002.

[9] P. Ogren, E. Fiorelli, and N. E. Leonard, "Cooperative control of mobile sensor networks: Adaptive gradient climbing in a distributed environment," IEEE Trans. Autom. Control, vol. 49, no. 8, pp. 1292-1302, 2004.

[10] D. M. Stipanovic, G. Inalhan, R. Teo, and C. J. Tomlin, "Decentralized overlapping control of a formation of unmanned aerial vehicles," $A u$ tomatica, vol. 40, no. 8, pp. 1285-1296, 2004.

[11] H. G. Tanner and A. Kumar, "Formation stabilization of multiple agents using decentralized navigation functions," Robot.: Sci. Syst. I, pp. 49-56, 2005.

[12] H. G. Tanner, A. Jadbabaie, and G. J. Pappas, "Stable flocking of mobile agents. Part II: Dynamics topology," in Proc. 42nd IEEE Conf. Decision Control, Maui, HI, 2003, vol. 2, pp. 2016-2021.

[13] S. S. Ge and Y. J. Cui, "New potential functions for mobile robot path planning," IEEE Trans. Robot. Autom., vol. 16, no. 5, pp. 615-620, Oct. 2000.

[14] J. Cortes, S. Martinez, and T. K. F. Bullo, "Coverage control for mobile sensing networks," IEEE Trans. Robot. Autom., vol. 20, no. 2, pp. 243-255, Apr. 2004.

[15] E. Rimon and D. E. Koditschek, "Robot navigation functions on manifolds with boundary," Adv. Appl. Math., vol. 11, no. 4, pp. 412-442, 1990.

[16] D. V. Dimarogonas and K. J. Kyriakopoulos, "Formation control and collision avoidance for multi-agent systems and a connection between formation infeasibility and flocking behavior," in Proc. 44th Conf. Decision Control/Eur. Control Conf., Seville, Spain, 2005, pp. 84-89.

[17] R. Olfati-Saber and R. M. Murray, "Distributed cooperative control of multiple vehicle formations using structural potential functions," presented at the 15th IFAC World Congr., Barcelona, Spain, 2002.

[18] D. V. Dimarogonas, S. G. Loizou, K. J. Kyriakopoulos, and M. M. Zavlanos, "A feedback stabilization and collision avoidance scheme for multiple independent non-point agents," Automatica, vol. 42, no. 2, pp. 229-243, 2006.

[19] D. Liberzon, Switching in Systems and Control. Cambridge, MA: Birkauser, 2003.

[20] R. Fierro and F. L. Lewis, "Control of a nonholonomic mobile robot: Backstepping kinematics into dynamics," in Proc. 34th IEEE Conf. Decision Control, New Orleans, LA, 1995, vol. 4, pp. 3805-3810.

[21] T. Fukao, H. Nakagawa, and N. Adachi, "Adaptive tracking control of a nonholonomic mobile robot," IEEE Trans. Robot. Autom., vol. 16, no. 5, pp. 609-615, Oct. 2000

[22] K. D. Do, Z. P. Jiang, and J. Pan, "A global output-feedback controller for simultaneous tracking and stabilization of unicycle-type mobile robots," IEEE Trans. Robot. Autom., vol. 20, no. 3, pp. 589-584, Jun. 2004.

[23] C. Samson, "Velocity and torque feedback control of a nonholonomic cart," in Advanced Robot Control, ser. Lecture Notes in Control and Information Sciences, C. Canudas de Wit, Ed. Berlin, Germany: Heidelberg, 1991, pp. 125-151.

[24] R. O. Saber and R. M. Murray, "Flocking with obstacle avoidance: Cooperation with limited communication in mobile networks," in Proc. 42nd IEEE Conf. Decision Control, Maui, HI, 2003, vol. 2, pp. 2022-2028.

[25] M. Krstic, I. Kanellakopoulos, and P. V. Kokotovic, Nonlinear and Adaptive Control Design. New York: Wiley, 1995.

[26] H. Khalil, Nonlinear Systems. Upper Saddle River, NJ: Prentice-Hall, 2002.

[27] H. G. Tanner and K. J. Kyriakopoulos, "Backstepping for nonsmooth systems," Automatica, vol. 39, no. 7, pp. 1259-1265, 2003.

[28] K. D. Do, Z. P. Jiang, and J. Pan, "Universal controllers for stabilization and tracking of underactuated ships," Syst. Control Lett., vol. 47, no. 4, pp. 299-317, 2002.

[29] K. D. Do and J. Pan, "Underactuated ships follow smooth paths with integral actions and without velocity measurements for feedback: Theory and experiments," IEEE Trans. Control Syst. Technol., vol. 14, no. 2, pp. 308-322, Mar. 2006 